\title{
Preconditioned mesenchymal stem cells treat myasthenia gravis in a humanized preclinical model
}

\author{
Muriel Sudres, ${ }^{1,2,3}$ Marie Maurer, ${ }^{1,2,3}$ Marieke Robinet, ${ }^{1,2,3}$ Jacky Bismuth, ${ }^{1,2,3}$ Frédérique Truffault, ${ }^{1,2,3}$ \\ Diane Girard, ${ }^{1,2,3}$ Nadine Dragin, ${ }^{1,2,3}$ Mohamed Attia, ${ }^{1,2,3}$ Elie Fadel, ${ }^{4}$ Nicola Santelmo, ${ }^{5}$ Camille Sicsic, ${ }^{6}$ \\ Talma Brenner, ${ }^{6}$ and Sonia Berrih-Aknin ${ }^{1,2,3}$ \\ 'Sorbonne Universités, UPMC Univ Paris 06, Paris, France. ${ }^{2}$ INSERM U974, Paris, France. ${ }^{3} \mathrm{AIM}$, Institute of Myology, \\ Paris, France. ${ }^{4}$ Centre Chirurgical Marie Lannelongue, Le Plessis Robinson, France. ${ }^{5}$ Hôpital Civil de Strasbourg, Strasbourg, \\ France. ${ }^{6}$ Department of Neurology, Agnes Ginges Center for human Neurogenetics, Hadassah Hebrew University Medical \\ Center, Jerusalem, Israel.
}

\begin{abstract}
Myasthenia gravis (MC) with anti-acetylcholine receptor (AChR) Abs is an autoimmune disease characterized by severe defects in immune regulation and thymic inflammation. Because mesenchymal stem cells (MSCs) display immunomodulatory features, we investigated whether and how in vitro-preconditioned human MSCs (cMSCs) could treat MG disease. We developed a new humanized preclinical model by subcutaneously grafting thymic MG fragments into immunodeficient NSG mice (NSG-MG model). Ninety percent of the animals displayed human antiAChR Abs in the serum, and $50 \%$ of the animals displayed MG-like symptoms that correlated with the loss of AChR at the muscle endplates. Interestingly, each mouse experiment recapitulated the MG features of each patient. We next demonstrated that CMSCs markedly improved MG, reducing the level of anti-AChR Abs in the serum and restoring AChR expression at the muscle endplate. Resting MSCs had a smaller effect. Finally, we showed that the underlying mechanisms involved (a) the inhibition of cell proliferation, (b) the inhibition of B cell-related and costimulatory molecules, and (c) the activation of the complement regulator DAF/CD55. In conclusion, this study shows that a preconditioning step promotes the therapeutic effects of MSCs via combined mechanisms, making cMSCs a promising strategy for treating MG and potentially other autoimmune diseases.
\end{abstract}

Conflict of interest: The authors have declared that no conflict of interest exists.

Submitted: July 19, 2016

Accepted: February 23, 2017

Published: April 6, 2017

Reference information: JCI Insight. 2017;2(7):e89665. https:// doi.org/10.1172/jci.insight.89665.

\section{Introduction}

Acquired myasthenia gravis (MG) is a rare autoimmune neuromuscular disease that is mediated by autoantibodies (autoAbs) directed against proteins of the neuromuscular junction (NMJ), which lead to fluctuating skeletal muscle weakness and fatigability. In $85 \%$ of MG patients, autoAbs specific for the nicotinic acetylcholine receptor $(\mathrm{AChR})$ are found in the serum. These Abs trigger the activation of the complement system, the accumulation of membrane attack complexes (MACs), the destruction of the postsynaptic muscle membrane, a reduction in the number of functional AChRs, and the disruption of neuromuscular transmission (1-3). The thymus, which is the site of $\mathrm{T}$ cell maturation and the establishment of central tolerance, is clearly involved in the pathogenesis of the disease. In AChR-seropositive MG patients, the thymus often displays structural and functional abnormalities, such as thymoma (15\%) or thymic follicular hyperplasia $(60 \%)$, which are characterized by the presence of ectopic germinal centers (GCs) (4). The hyperplastic thymus contains all of the components of the anti-AChR immune response: the autoantigen, autoreactive $\mathrm{T}$ cells, and autoAb-producing $\mathrm{B}$ cells. The MG thymus proinflammatory environment is suspected to induce immune dysregulation that promotes autoimmune reactions $(5,6)$. Thymectomy, which is performed primarily in early-onset MG patients, represents 1 of the 4 therapeutic options, in addition to (a) acetylcholinesterase inhibitors (a symptomatic therapy that improves neuromuscular transmission), (b) steroids and immunosuppressive agents (generally used for long-term therapy), and (c) plasmapheresis and intravenous immunoglobulins (used to treat acute MG exacerbation). Despite those therapeutic options, MG remains debilitating and problematic to stabilize. Furthermore, steroids and immunosuppressive drugs can cause severe side effects, as such drugs are long-term treatments (7). Thus, more efficient and less toxic treatments must be developed. 
Table 1. Details for the myasthenia gravis (MG) patients included in the study

\begin{tabular}{|c|c|c|c|c|c|c|c|c|c|c|c|}
\hline \multicolumn{3}{|c|}{ Patients } & \multicolumn{4}{|c|}{ MG features } & \multicolumn{5}{|c|}{ Treatments } \\
\hline & Age & Sex & Score ${ }^{A}$ & Grade $^{B}$ & Ab titers & GC & $C x$ & IS & AA & IVIg & PP \\
\hline 1 & 15 & $\mathrm{~F}$ & 54 & na & $>100$ & ++ & no & no & yes & no & no \\
\hline 2 & 36 & $\mathrm{~F}$ & 85 & Ila & $>100$ & + & no & no & yes & no & no \\
\hline 3 & 13 & $\mathrm{~F}$ & 55 & Illa & $>100$ & - & yes & no & yes & no & yes \\
\hline 4 & 28 & $M$ & 98 & na & $>100$ & + & yes & no & yes & no & no \\
\hline 5 & 18 & $\mathrm{~F}$ & 66 & Ilb & $>100$ & ++ & no & no & yes & no & no \\
\hline 6 & 32 & $\mathrm{~F}$ & 65 & IIb & $>100$ & +++ & no & no & yes & yes & no \\
\hline 7 & 24 & $\mathrm{~F}$ & 90 & lb & 99.3 & ++ & no & no & yes & no & no \\
\hline 8 & 20 & $M$ & 78 & Ila & 87.6 & + & no & no & yes & no & no \\
\hline 9 & 18 & $\mathrm{~F}$ & 65 & Ila & 57.5 & + & no & no & yes & yes & no \\
\hline 10 & 15 & $\mathrm{~F}$ & 95 & la & 15 & na & yes & no & yes & yes & no \\
\hline 11 & 19 & $\mathrm{~F}$ & 90 & Ib & 4.23 & ++ & yes & yes & yes & no & no \\
\hline 12 & 27 & $\mathrm{~F}$ & 75 & Ila & 1.14 & + & no & no & no & no & no \\
\hline 13 & 16 & $M$ & 74 & Ila & 0.92 & - & no & no & yes & yes & no \\
\hline 14 & 22 & $M$ & 85 & na & $<0.5$ & - & no & no & yes & no & no \\
\hline 15 & 29 & $\mathrm{~F}$ & na & na & $<0.5$ & - & no & no & yes & no & no \\
\hline 16 & 20 & $\mathrm{~F}$ & na & na & $<0.5$ & - & no & no & yes & no & no \\
\hline 17 & 12 & $M$ & 95 & la & $<0.5$ & na & yes & no & yes & yes & no \\
\hline
\end{tabular}

ACarches hospital scores (65). ${ }^{B}$ Myasthenia Gravis Foundation of America (MGFA) grade (66). The average age is $21.7 \pm 6.9$ years. The sex ratio is 1:4.25 (M:F). Patients with anti-acetylcholine receptor (AChR) Ab titers below $0.5 \mathrm{nmol} / \mathrm{I}$ are considered to be seronegative. Patients with AChR Ab titers above $50 \mathrm{nmol} / \mathrm{l}$ are considered to be highly seropositive. Low seropositive MG patients have AChR Ab titers between 0.5 and 20 nmol/l. Patients with many germinal centers (GCs) in the thymus are noted as +++, patients with some GCs are noted as ++ , patients with few GCs are noted as + and patients with no GCs are noted as -. Cx, corticoids; IS, immunosuppressive agent; AA, anti-acetylcholinesterase; PP, plasmapheresis. As expected, patients with positive or high anti-AChR Abs display GCs in their thymus (67). The only exception is patient 3, who was treated with corticosteroids, which have an effect on GCs but not on anti-AChR Ab titers (67).

To this end, relevant animal models that mimic the human disease are crucial. Current experimental MG models are induced in rodents using 2 strategies. First, AChR immunization generates experimental autoimmune MG (EAMG), but this model presents an inflammatory bias and does not reproduce thymus abnormalities (8). Second, MG thymus tissue or cells can be grafted into immunodeficient mice, but this model does not reproduce clinical weakness and human cells could not be detected $(9,10)$.

Mesenchymal stem cells (MSCs) are multipotent progenitor cells that are found in various organs, such as the bone marrow and adipose tissue. MSCs were shown to display regenerative and trophic features, as well as antiinflammatory and immunosuppressive capabilities. MSCs can exert their immunosuppressive effect on both innate and adaptive immune cells (11) via several mechanisms that are currently well defined but remain under investigation (12). Thus, MSCs are a promising tool for treating autoimmune diseases. MSCs were shown to have favorable effects in experimental models of multiple sclerosis, systemic lupus erythematosus, and rheumatoid arthritis (13), as well as in the EAMG model $(14,15)$.

MSCs are currently being investigated in more than 700 clinical trials, including about 60 trials for autoimmune disorders, such as multiple sclerosis, Crohn's disease, rheumatoid arthritis, and systemic lupus erythematous (ClinicalTrial.gov). Both autologous and allogeneic MSCs are being tested. To our knowledge, only resting cells are being used in those therapeutic assays. However, it appears that the pretreatment of MSCs could improve their immunosuppressive properties, as the pretreatment of MSCs with inflammatory cytokines, such as IFN- $\gamma$, TNF- $\alpha$, and IL-17, has been shown to promote their immunosuppressive potential $(16,17)$. In our previous report, we showed that supernatants from conditioned MSCs but not resting MSCs were able to inhibit the proliferation of peripheral blood cells (18).

In this study, we aimed to develop a new humanized mouse model that recapitulates all of the features of the human MG disease to evaluate the therapeutic potential of human MSCs and determine whether and how MSC priming could result in enhanced efficacy in comparison with resting MSCs (rMSCs). 


\section{Results}

$M G$ thymus transplantation induced $M G$ features. To develop a relevant humanized preclinical model, thymus fragments from MG patients were transplanted subcutaneously into immunodeficient NSG (NOD-scid IL-2R $\gamma^{\text {null }}$ ) mice. Table 1 summarizes the clinical details of the MG patients. Altogether, we performed 17 thymic grafts from MG patients and 11 thymic grafts from non-MG (CTRL) patients. Among the MG patients, 4 thymuses were from AChR-seronegative patients, 4 thymuses were from AChR-seropositive patients displaying low $\mathrm{Ab}$ titers (MG low, from 1 to $20 \mathrm{nM}$ ), and 9 thymuses were from AChR-seropositive patients displaying high $\mathrm{Ab}$ titers (MG high, above $20 \mathrm{nM}$ ). Unsurprisingly, women represented more than $70 \%$ of the patients. The average age was $21.7 \pm 6.9$ years (range from 12 to 36 years). Sixteen patients were treated with acetylcholinesterase inhibitors ( 4 of those patients also received corticosteroids and 1 of those patients received corticosteroids plus an immunosuppressive agent). One patient received no treatment at all.

Human AChR-specific Abs were detected in the serum of mice receiving thymus fragments from AChR-seropositive MG patients but not in the serum of mice receiving thymus fragments from AChRseronegative patients or CTRL subjects. Figure 1A shows the levels of AChR-specific Abs in the different experimental groups. The kinetics of anti-AChR Abs are shown in Supplemental Figure 1A (supplemental material available online with this article; https://doi.org/10.1172/jci.insight.89665DS1) that displays the mean value of all experiments as well as some individual experiments (Supplemental Figure 1, B-E).

In both the MG low and MG high groups, $50 \%$ of the mice displayed clinical signs (Figure 1B), such as abnormal or reduced movement and sometimes death. The first symptoms occurred 2 weeks after transplantation and continued to develop during the next month (mean times to occurrence of $35.7 \pm 11.6$ days and 27.1 \pm 11.5 days for MG high and MG low mice, respectively). We observed no clinical signs in mice grafted with thymus fragments from AChR-seronegative patients or CTRL patients. Interestingly, symptom severity was fairly similar in the MG low and MG high groups (Supplemental Figure 1F).

To investigate the link between MG symptoms and NMJ abnormalities, we quantified AChR contents at the diaphragmatic endplates. We observed an AChR loss in mice grafted with thymus fragments from both low- and high-titer AChR-seropositive patients in comparison with mice grafted with CTRL fragments $(22.9 \%$ and $22.4 \%$ reduction, respectively) (Figure $1 \mathrm{C})$, highlighting the lack of correlation between the level of Abs and the pathogenic effects on AChR expression. Furthermore, we observed that endplate AChR loss inversely correlated with MG clinical scores (Figure 1D). Thus, similar to the human disease, MG severity was not correlated with anti-AChR Abs but was correlated with AChR expression loss in the muscle endplates.

Additionally, we observed that the mean mouse anti-human AChR level correlated with the level observed in the patient sera and that the mean mouse global score correlated with the patient score at the time of thymectomy (Figure 1, E and F, respectively); in other words, each mouse experiment recapitulated the features of each MG patient. Furthermore, in both mice and humans, AChR-specific Ab levels failed to correlate with clinical scores (Figure 1, G and $\mathrm{H}$, respectively). Of note, in Figure 1F we excluded the patients treated with corticosteroids, as such patients behave differently from untreated patients, likely because of the discontinuation of the treatment in the mice after grafting (Supplemental Figure 1G). Indeed some patients (MG4 and MG11) had a good MG score under treatment but had a significant clinical score in mice.

Here, we demonstrate that MG thymus tissue is sufficient to induce MG symptoms in mice. As a result, our NSG-MG model truly mimicked the human disease.

Xenogeneic thymus fate. We then analyzed the fate of the human thymus tissues in the mice. We first noticed that new vessels developed around the thymus fragments in nearly all of the mice, in both the MG and CTRL groups (Figure 2A). Six to 8 weeks after grafting, we performed histological sections and observed a preserved thymic architecture, with distinguishable cortical and medullary areas in the lobules (Figure 2B). Immunofluorescence labeling showed many epithelial cells and abundant connective tissue (Figure 2C), as well as T cells (Figure 2D). No significant difference in the expression of CD4 and CD8 mRNA was observed between MG and CTRL thymuses (Figure 2, E and F). These data indicate that the human thymus tissues were preserved ultrastructurally for at least 6 to 8 weeks after grafting.

We next analyzed the transcripts of relevant genes that are usually involved in the physiopathology of the disease, starting with B cell activation- and maturation-related genes. Indeed, in AChR MG patients, the thymus displays ectopic GCs (19) that contain a large number of B cells (4). As expected, we observed the overexpression of the following genes in MG thymuses in comparison with CTRL thymuses: the B cell 
A

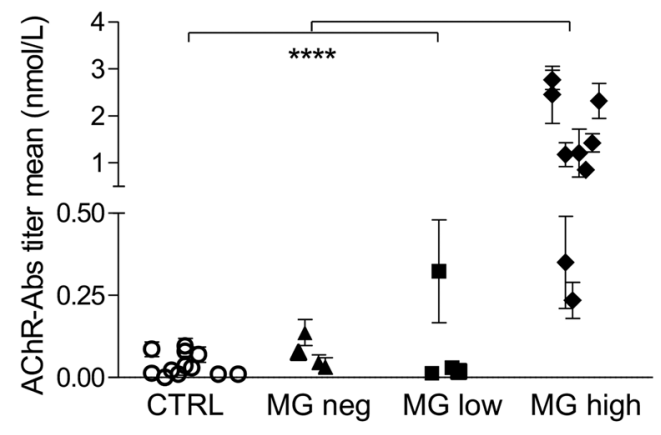

C

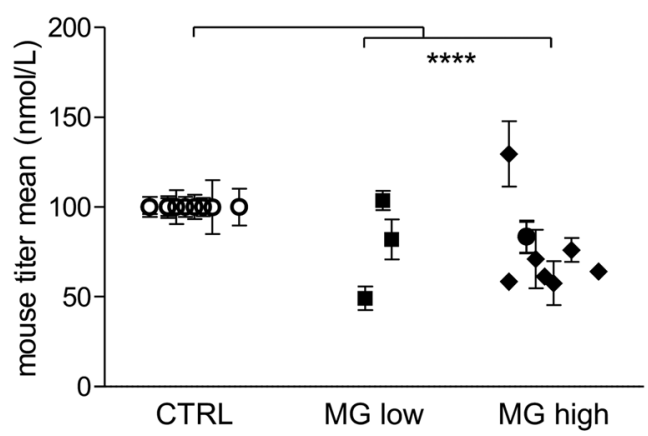

E

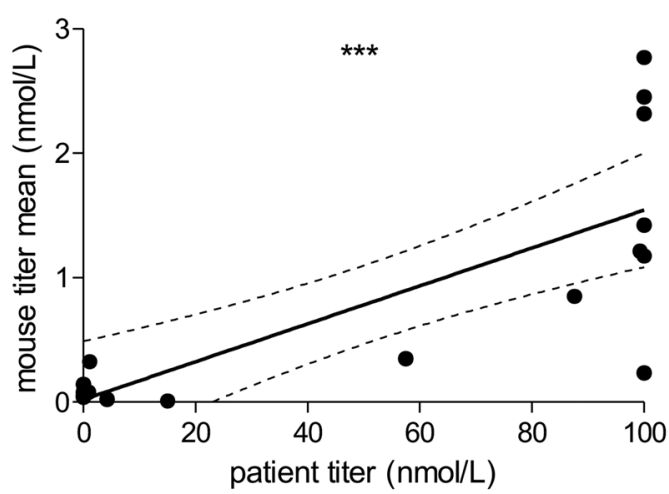

G

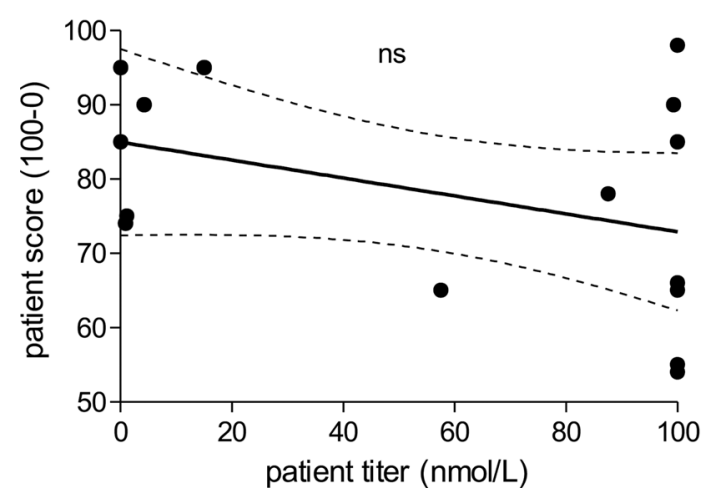

B

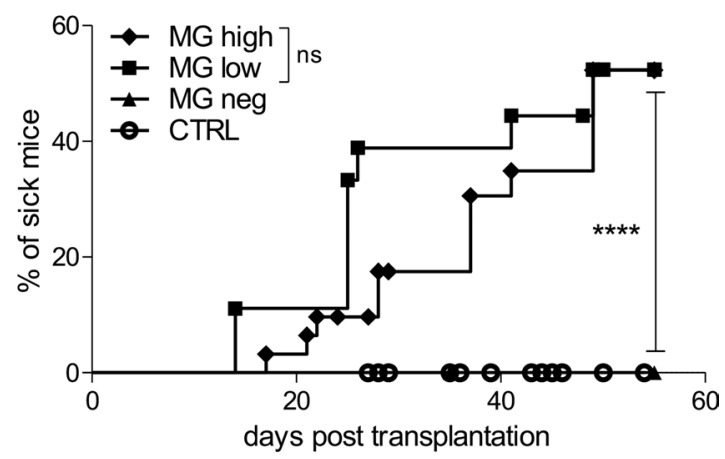

D

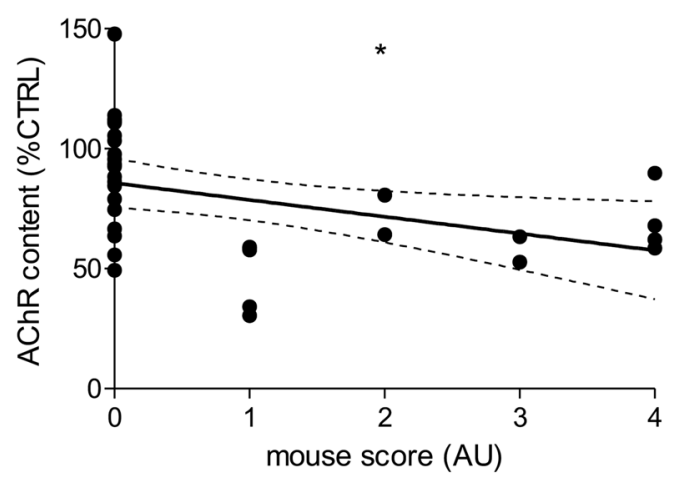

F

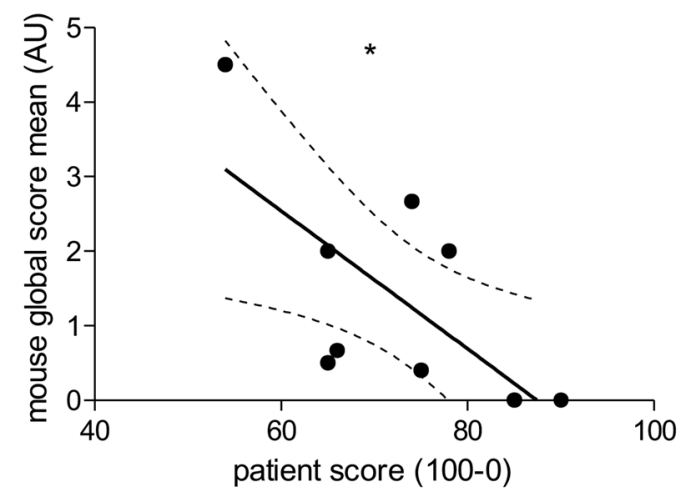

H

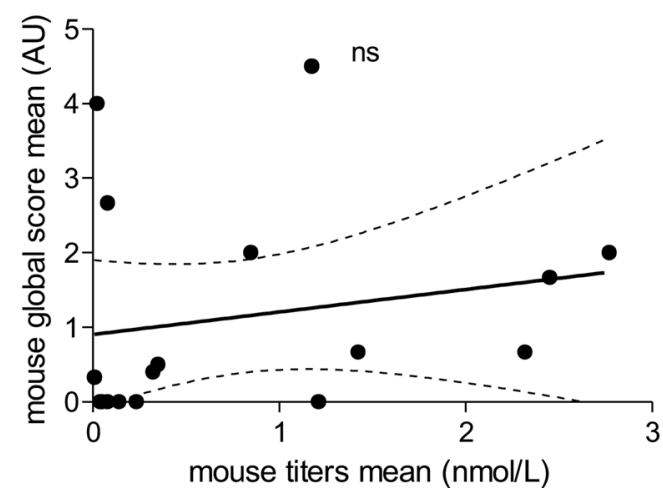

Figure 1. Characterization of the novel NSG-MG model. (A) Human acetylcholine receptor-specific (AChR-specific) Abs were measured in mouse serum in the 3 myasthenia gravis (MG) groups and the control (CTRL) group. Each symbol represents the mean value \pm SEM of human anti-AChR Ab titers measured in mice for each experiment (CTRL group, 12 grafts, 51 mice; MG neg group, 4 grafts, 14 mice; MG low, 4 grafts 16 mice, MG high group, 9 grafts, 31 mice). (B) The mice displayed MG-like clinical symptoms. The Kaplan-Meyer curve shows the occurrence of the disease (score $>1)$ in the high-titer anti-AChR seropositive MG group $(n=31)$, in the low-titer seropositive MG group $(n=18)$, in the seronegative MG group $(n=14)$, and in the CTRL group 
$(n=51)$. (C) The endplate AChR content of the diaphragmatic muscle (measured by ${ }^{125}$ - $\alpha$-bungarotoxin labeling) was diminished in the MG groups. The cpm data are normalized using the mean cpm values of the CTRL group (levels set at 100\%). Each symbol represents the cpm value mean \pm SEM of each graft experiment (CTRL group, 10 grafts, 37 mice; MG low group, 3 grafts, 10 mice; MG high group, 8 grafts, 25 mice). (D) Endplate AChR loss correlated with MG severity. Each symbol represents 1 mouse from the MG groups (seronegative and seropositive). (E) Patient Ab titers correlated with mouse Ab titers. Each symbol represents the AChR-specific Ab titer measured in $1 \mathrm{MG}$ patient and the corresponding mean Ab titer value measured in mice for each experiment $(P<0.0003)$. (F) The patient score correlated with the mouse score. Each symbol represents the score of $1 \mathrm{MG}$ patient (untreated by corticosteroids) and the corresponding mean score obtained in mice for each experiment $(P<0.03)$. $(\mathbf{G}$ and $\mathbf{H})$ In humans $(\mathbf{C})$ and mice $(\mathbf{H})$, Ab titers did not correlate with clinical scores. $P$ values were determined using the 1-way ANOVA test (A and $\mathbf{C}$ ) or the log-rank (Mantel-Cox) test (B). (D-H) $R$ and $P$ values were determined using the linear regression test. ${ }^{*} P<0.05$; ${ }^{* *} P<0.001 ;{ }^{* * *} P<0.0001$. ns, not significant.

marker CD20 (Figure 2G) and BAFF, which is involved in B cell differentiation and survival (20, 21) (Figure $2 \mathrm{H}$ ). Conversely, IRF4, which is a pivotal regulator of B cell fate dynamics (22), exhibits lower expression in MG thymuses than in CRTL thymuses (Figure 2I). In addition, we were able to detect some GCs in the xenogeneic thymuses of mice displaying the most obvious clinical signs via immunofluorescence (Figure 2J). These data strongly suggest that autoimmune GCs are maintained in vivo for several weeks.

As an inflammatory environment is likely to promote autoimmunity, including MG (23), we then analyzed proinflammatory genes. We observed signs of inflammation, with significant overexpression of IL-2 (Figure 2K), IL-6 (Figure 2L), IL-17 (Figure 2M), TNF- $\alpha$ (Figure 2N), and INF- $\gamma$ mRNA (Figure 2O) in MG thymuses in comparison with CRTL thymuses. These findings are very similar to the findings described for MG thymuses investigated immediately after thymectomy (5). Of note, MG low and MG high patients behaved similarly and thus these groups were combined in a single MG group.

Thus, the human MG thymuses retained active pathogenic features, including GCs and high inflammatory cytokine levels, in the NSG-MG model for at least 2 months after grafting.

MG T cells but not MG B cells home to the spleen. Because neovascularization was observed around the xenogeneic tissues, we hypothesized that human cells were able to exit from the thymus, and we investigated the ability of those cells to survive in the mouse environment. In both groups, 6 to 8 weeks after transplantation, human cells (CD45-positive cells) were detected in the spleen, in the blood, and to a lesser extent, in the bone marrow (Figure 3A). Interestingly, the MG group displayed more human cells than the CTRL group, especially in the spleen (Figure 3B, $23.0 \% \pm 3.0 \%$ and $10.7 \% \pm 2.7 \%$ of CD 45 -expressing cells, respectively). Immunohistochemical studies of the spleens of the mice showed fewer human cells (lamin A/C-positive cells) in mice grafted with control thymus fragments (Figure 3C) than in mice grafted with MG thymus fragments (Figure 3D), confirming that a larger number of cells migrate from the MG thymus than from the normal thymus. In parallel, we noticed larger spleens in mice grafted with MG thymuses in comparison with CTRL (Figure 3E).

Furthermore, we observed that the human cells were organized in more or less compact clusters in the mouse spleen (Supplemental Figure 2, A and B). Thus, the human cells were able to circulate and home to mouse lymphoid organs.

Because the MG thymus contains a large number of B cells, we wondered whether MG B cells alone or all MG thymocytes could explain this high level of homing. An analysis of the percentage of human cells among the total cells in the mouse spleen showed that CD4 single positive (CD4 ${ }^{+} \mathrm{SP}$ ) (Figure $4 \mathrm{~A}$ ), $\mathrm{CD}^{+} \mathrm{SP}$ (Figure 4B), and CD19+ (Figure 4C) cells were more numerous in the spleens of the MG group than in the spleens of the CTRL group. However, an analysis of the percentage of human cells among CD45-positive cells showed that the mean percentage of B cells was less than $10 \%$ (Figure 4D) and was surprisingly similar in the MG and CTRL groups. Because we observed an overexpression of CD20 mRNA in the thymus, we hypothesized that B cells within GCs migrate poorly to the periphery. When performing an analysis of CD45-positive cells, we also noticed that in both the MG and CTRL groups, most of the CD45-positive cells were $\mathrm{CD}^{+} \mathrm{SP}$ cells $\left(50 \%\right.$ to $60 \%$ ) and $\mathrm{CD} 8^{+} \mathrm{SP}$ cells (20\% to $30 \%$ ), indicating that mature human $\mathrm{T}$ lymphocytes home to the periphery and suggesting that both MG and CTRL thymocytes end their differentiation in vivo (data not shown).

Thus, the human cells were able to survive in grafted animals for several weeks within the xenogeneic thymus and the mouse lymphoid organs.

To summarize, we succeeded in developing a robust and humanized MG preclinical model that exhibits MG-like symptoms, anti-AChR Abs, and the loss of AChR at the muscle endplates, and we demonstrated that human thymic cells migrated to the spleen. We then evaluated MSCs as a therapeutic strategy for treating $\mathrm{MG}$. 
A

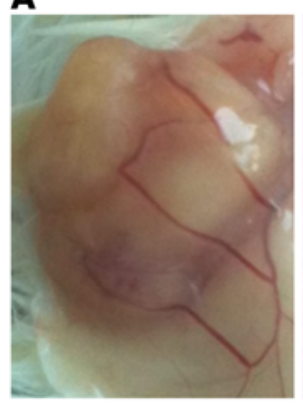

E

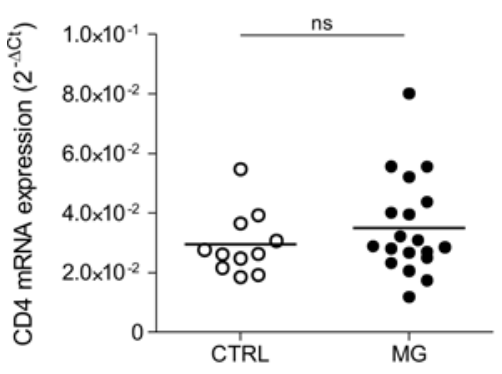

H

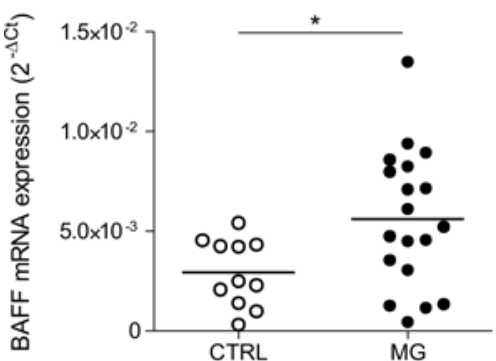

$\mathbf{K}$

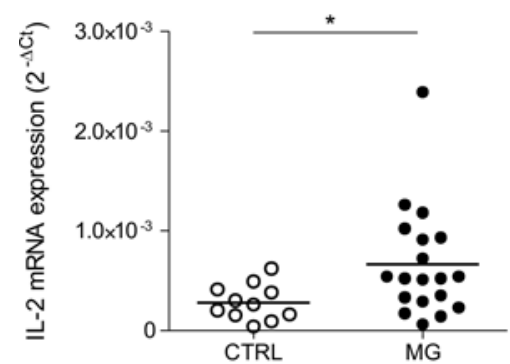

$\mathbf{N}$

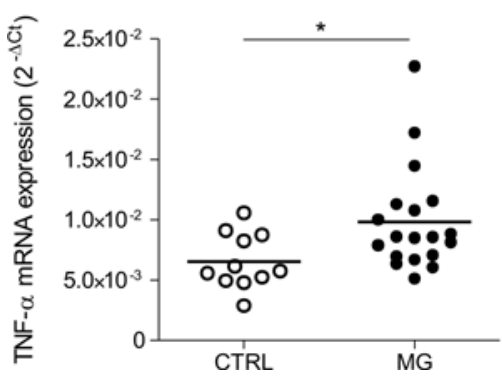

B

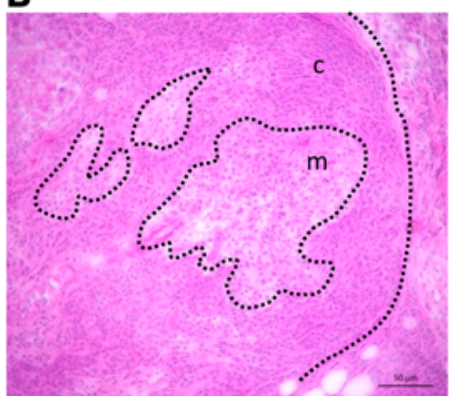

$\mathbf{F}$
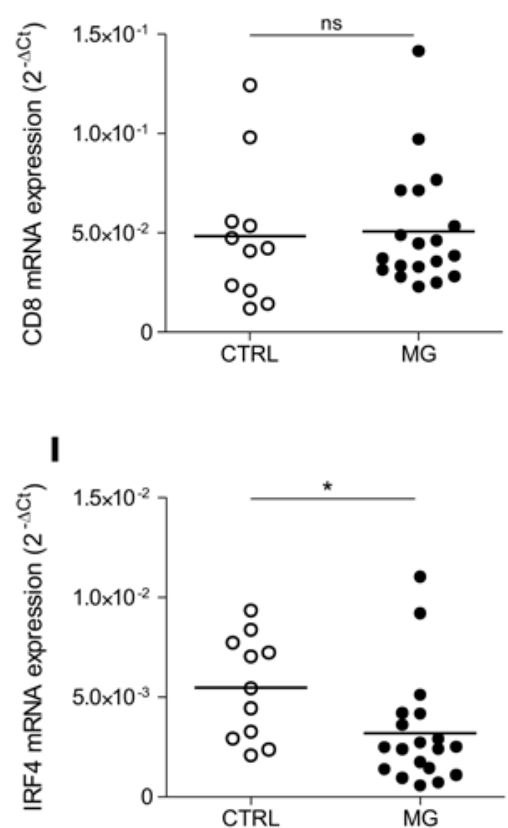

$\mathbf{L}$

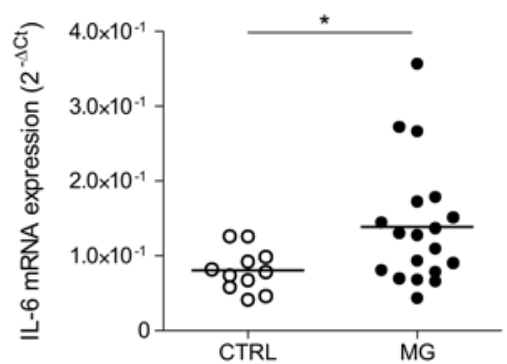

0

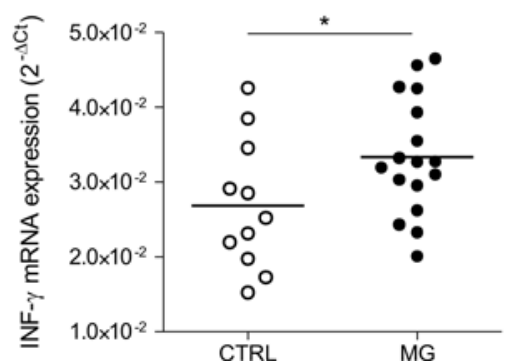

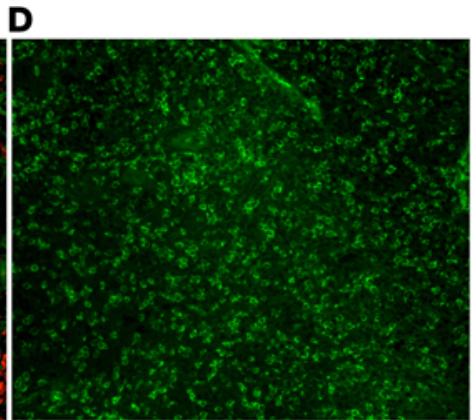

CD4 and CD8
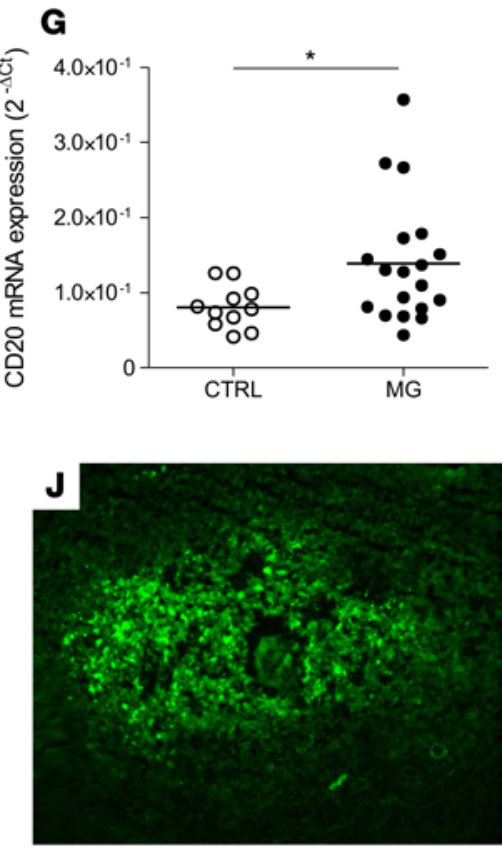

CD21 (GC)

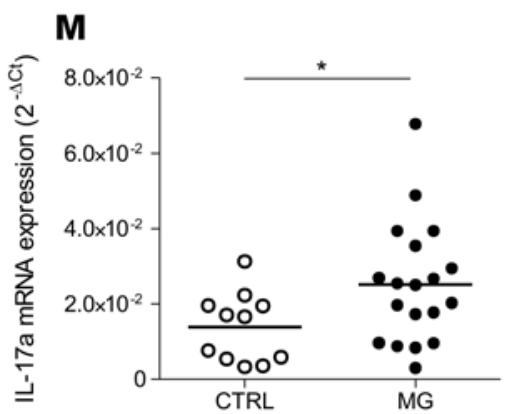


Figure 2. Xenogeneic thymus fate. The human thymus is structurally preserved 2 months after grafting. (A) Image of thymic fragments in the mouse's back 2 months after grafting. (B) H\&E coloration of a thymic section. c, cortex; m, medulla. Scale bar: $50 \mu \mathrm{m}$. (C) Keratin (in red) and fibronectin (in green) labeling of a thymic section. Original magnification, $\times 300$. (D) CD4 and CD8 labeling of a thymic section. Original magnification, $\times 300 .(E$ and $\mathbf{F})$ CD4 (E) and CD8 (F) mRNA expression were analyzed via real-time PCR (qPCR). (G-I) CD20 (G), BAFF (H), and IRF4 (I) mRNA expression levels were analyzed via qPCR. (J) CD21 labeling (showing germinal center [GC]) in a thymic section from a grafted mouse. Original magnification, $\times 600$. (K-0) IL-2 (K), IL-6 (L), IL-17a (M), TNF- $\alpha(\mathbf{N})$, and IFN- $\gamma(\mathbf{O})$ mRNA expression levels were analyzed via qPCR. Each symbol represents the $2^{-\Delta c t}$ value of 1 mouse, and the bars represent the mean values in the control (CTRL) and myasthenia gravis (MG) groups. ${ }^{*} P<0.05$ by the Mann Whitney $t$ test; $C T R L, n=11$ (4 experiments); MG, $n=19$ ( 6 experiments). ns, not significant.

MSC treatment reduced MG features. We demonstrated previously that supernatants from MSCs obtained after coculture with peripheral blood mononuclear cells (PBMCs) suppressed the proliferation of $\mathrm{T}$ cells much more efficiently than supernatants from MSCs alone (13). We therefore compared the therapeutic efficacy of preconditioned MSCs (cMSCs) versus rMSCs in the NSG-MG model. Our MSC in vitro priming procedure consisted of a 3-day coculture (via a Transwell membrane) with healthy allogeneic PBMCs, and the MSCs were administered at the end of the second week after grafting on average.

In the MG group, the first clinical signs occurred during the second week after transplantation, and $46.1 \%$ of the animals were sick in the 8 th week after transplantation (Figure 5A). Disease occurrence was slightly lower in mice injected with rMSCs (39.1\%) than in untreated mice and was delayed by approximately 2 weeks. MG severity in the rMSC group did not differ significantly from the untreated MG group (Figure 5B, $0.78 \pm 0.28$ and $1.00 \pm 0.27$, respectively). However, cMSC treatment significantly decreased (4-fold) and delayed (nearly 1 month) MG occurrence (7.1\%) (Figure 5A), and improved clinical symptoms (Figure 5B). Mice that received either rMSCs or cMSCs gained body weight during the graft experiments, in contrast to untreated mice. The effects of MSCs on body weight were clear 3 weeks after treatment (Supplemental Figure 3). Thus, MSC treatment, especially treatment with cMSCs, improved MG clinical signs.

Whereas anti-AChR Ab levels increased in the serum of the MG group during the grafting experiments, rMSC- and cMSC-treated mice displayed stabilized and reduced titers, respectively (Figure 5C). Furthermore, MSC treatments had a protective effect on AChR at the NMJ (Figure 5D), although cMSCs were more efficient than rMSCs. Indeed, if rMSC injection treatment resulted in limited endplate AChR loss compared with the MG group (10.0\% vs. $19.8 \%$ reduction, respectively), cMSC injection abrogated that effect, as the mean AChR expression in this group did not differ from that of the control group. Altogether, cMSC treatment was able to reduce anti-AChR Abs in the serum of the mice, to increase AChR expression at the diaphragmatic NMJ, and to subsequently improve the global clinical status of the mice.

Mechanism of action of $c M S C$-mediated therapy. Because cMSC therapy improved MG, we next addressed the related mechanism. To this end, we performed a global assessment of genes involved in activation, differentiation, and migration in the thymus and spleen. Altogether, 57 genes were successfully analyzed, including transcription factors, signaling molecules, chemokines and chemokine receptors, accessory molecules, cycle and activation molecules, and cell subset-associated molecules. The study also included 3 housekeeping genes. Supplemental Table 1 shows the changes in gene transcripts in the MG group compared with the CTRL group, as well as in MSC-treated groups compared with the MG group in the thymus. Among the 57 genes, more than 20 were deregulated in the MG thymus compared with controls, and most of them were overexpressed. This includes Th1 and Th17 signature (IL-17A, IL-6, IFN- $\gamma$, and TNF- $\alpha$ ), B cell-related molecules (BAFF, APRIL, CD20, and BCL6), and accessory molecules (CD40 and PD-L1). cMSC treatment induced a normalization of the expression of several of these genes, namely BAFF, CD20, TNF- $\alpha$, CD40, and PDL1, although no effects were observed on Th17-related genes. Interestingly, only 1 gene (DAF/CD55) was significantly increased in the MSC-treated group. The effects of rMSCs were minimal; only 3 genes (CD40, CD40L, and TNF- $\alpha$ ) showed changes with a $P$ value between $5 \%$ and 10\%. Figure 6 illustrates the results obtained for TNF- $\alpha$ (Figure 6A), BAFF (Figure 6B), CD40L (Figure 6C), CD40 (Figure 6D), PD-L1 (Figure 6E), and CD55 (Figure 6F) in both MSC-treated groups compared with the untreated group.

We then focused on the mki67 gene (encoding a nuclear protein that is associated with cellular proliferation). Both in the xenogeneic thymus (Figure 7A) and in the spleen (Figure 7B), we observed that the transcripts of the mki67 gene were diminished in the cMSC group, but not in the rMSC group, in comparison with the MG group. Furthermore, we observed a clear correlation between mki67 mRNA levels and KI-67 protein levels in the spleen (Figure 7C). Images of KI-67 labeling in spleen sections showed that the fluorescence intensity was higher in the MG group (Figure 7D) than in the cMSC group (Figure 7E). We next wondered 
A

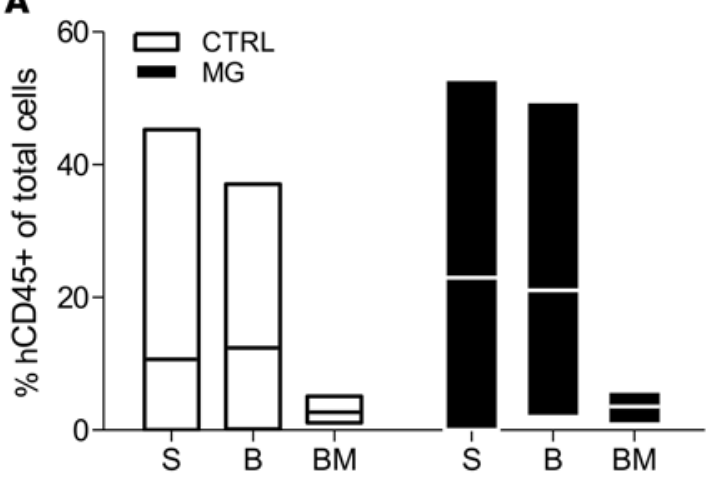

B

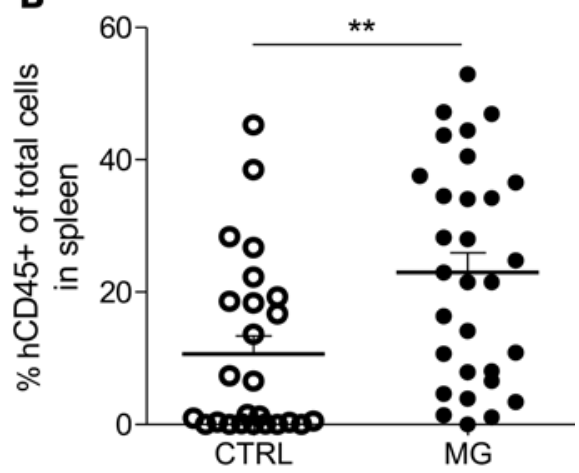

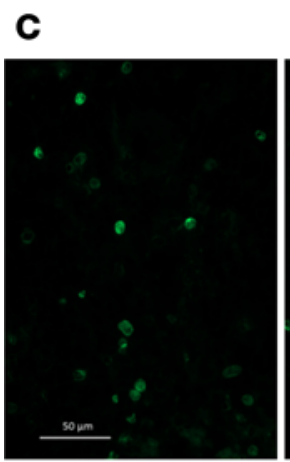

CTRL

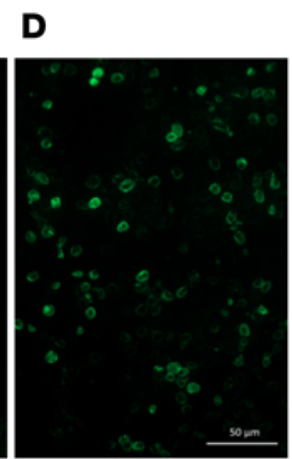

MG

LaminA/C (human nucleus)

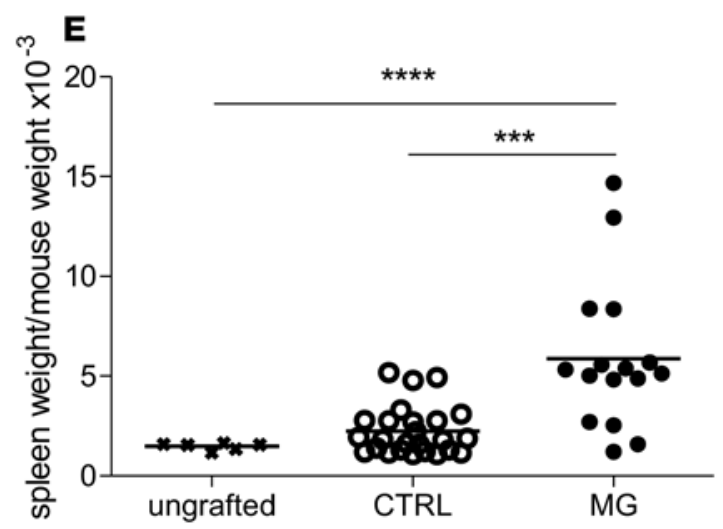

Figure 3. Human cells home to mouse lymphoid organs. (A) FACS analysis of the expression of CD45-positive cells in the spleen (S) (control [CTRL], $n=25$; myasthenia gravis [MG], $n=26,6$ to 7 experiments), in the blood (B) (CTRL, $n=5$; MG, $n=11,2$ to 4 experiments) and in the bone marrow (BM) (CTRL, $n=7 ;$ MG, $n=5,2$ experiments) of grafted animals. The floating bars represent the minimum to maximum percentage of CD45-expressing cells in each group, with lines set at the mean value. (B) FACS analysis of the expression of CD45-positive cells in the spleen. Each symbol represents the percentage of CD45-expressing cells in each mouse, and the bars represent the mean for each group. (C and D) Immunohistochemistry was performed on spleen sections to visualize human cells (lamin A/C-positive cells, in green) in mice grafted with control (C) or MC thymus fragments (D). Control sections with the secondary Ab were negative (not shown). Scale bars: $50 \mu \mathrm{m}$. (E) Spleen weights of grafted mice (CTRL, $n=25 ; M G, n=16$ ) in comparison with ungrafted mice $(n=6)$. The bars represent the mean value for each group. ${ }^{* *} P<0.01 ;{ }^{* *} P<0.001$; ${ }^{* * *} P<0.0001$ by Mann-Whitney (B) and 1-way ANOVA tests $(\mathbf{E})$.

whether a specific cell population was affected by the decreased proliferation. We observed that MSC treatment did not modify the T and B cell percentage in the spleen of MG thymus-grafted animals (Supplemental Figure 4). Even the absolute numbers of $\mathrm{T}$ and $\mathrm{B}$ cells did not significantly differ between untreated and MSCtreated groups (Supplemental Figure 4F). These results are in agreement with the PCR analysis performed in the spleen that did not show CD4, CD8, or CD19 mRNA differential expression between rMSCs and cMSCs treated in comparison with the CTRL (not shown). Interestingly, the PCR analysis performed in the thymus showed a tendency for a decrease in CD20 mRNA expression in the cMSC group compared with the MG group, compatible with a decrease in B cell proliferation in the thymus (Supplemental Figure 5).

These data suggest that one of the mechanisms of action of cMSCs in our NSG-MG model relies on the inhibition of cellular proliferation, probably including thymic B cells. In addition, our results demonstrate that the effects of cMSCs were more marked than those of rMSCs.

Altogether, our data indicate (a) that in addition to clinical improvements, cMSCs were able to modulate the transcription of genes involved in MG, (b) that cellular proliferation inhibition, TNF pathway inhibition, costimulatory molecule inhibition, and DAF promotion could represent non-mutually exclusive mechanisms of action of cMSCs, and (c) that cMSCs likely exerted their immune suppressive effects in the xenogeneic thymus rather than in the periphery because the main changes in gene expression were seen in the xenogeneic thymus and not in the spleen. 
A

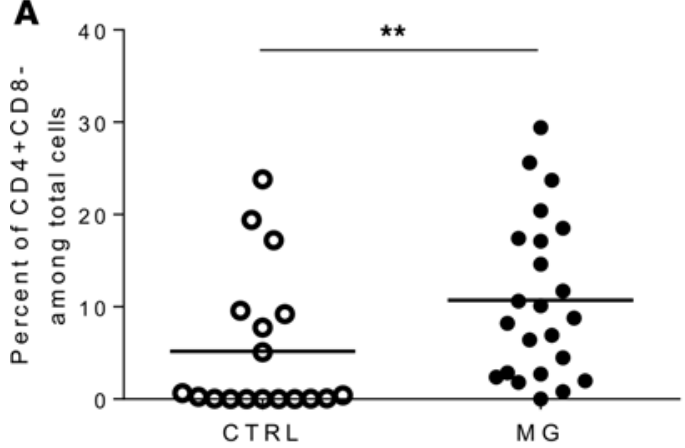

C

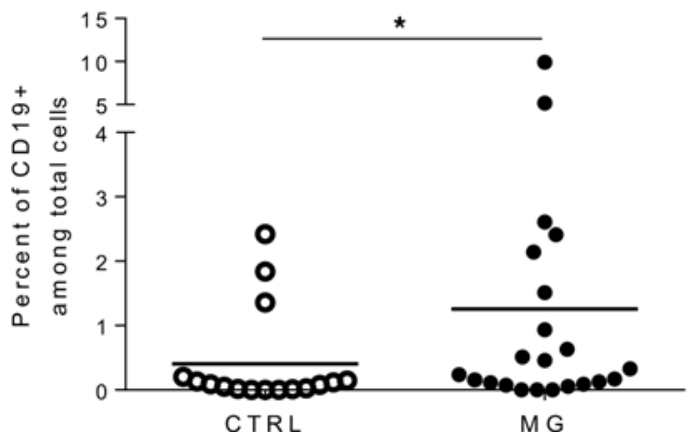

B

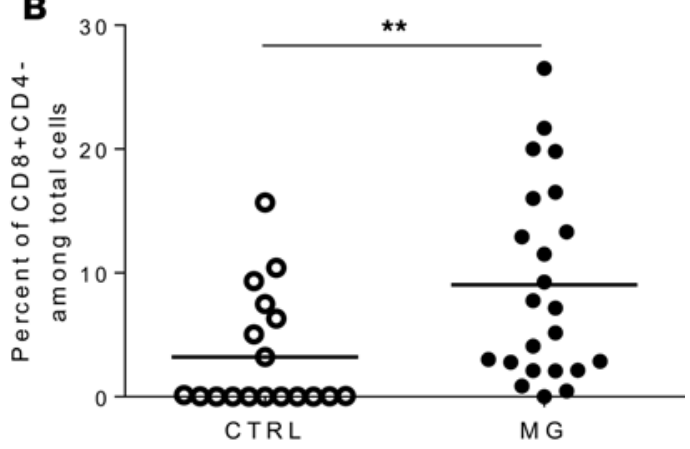

D

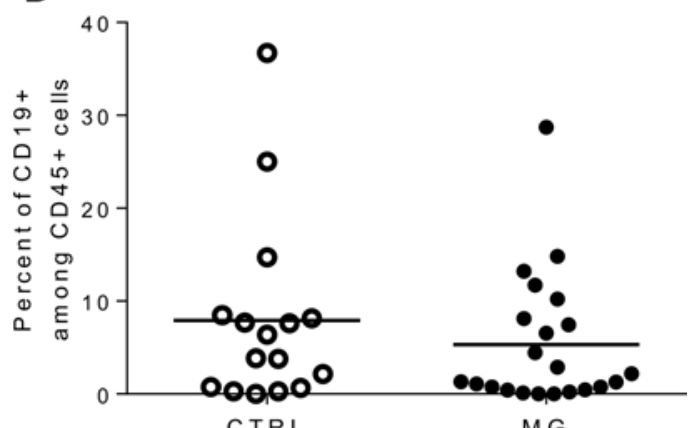

Figure 4. Human lymphocytes in the spleens of NSC mice. FACS analysis of the expression of CD4 single positive (CD4SP) (A), CD8SP (B), and CD19 (C and $\mathbf{D}$ ) among all splenocytes (A-C) or among CD45-expressing cells (D) in the spleens of grafted animals. For the CD4SP and CD8SP analyses, control (CTRL), $n=18$ and myasthenia gravis (MC), $n=23$. For the CD19 analyses, CTRL, $n=16$, and MG, $n=22$. Each symbol represents the percentage of $C D$-expressing cells for 1 mouse, and the bars represent the mean values. Four to 6 experiments are included. ${ }^{*} P<0.05,{ }^{* *} P<0.01$ by the Mann-Whitney $t$ test.

\section{Discussion}

The aim of this study was to evaluate the therapeutic efficacy of MSCs for MG. To this end, we developed a new experimental humanized model that mimics patient features. Our main results are as follows: (a) the humanized NSG-MG model appeared to be very robust and reproducible, mirroring MG disease with thymic pathology; (b) cMSCs were much more efficient than rMSCs as therapy for MG; and (c) the mechanisms of cMSCs involved the inhibition of proliferation, reduced expression of several molecules of the TNF pathway and costimulatory molecules, and the stimulation of the CD55 complement inhibitor.

NSG-MG: A new preclinical MG model for testing human therapies. Among 48 mice transplanted with MG thymuses from AChR-seropositive patients, 44 mice displayed AChR-specific Abs in the serum and 22 mice displayed obvious clinical signs correlated with AChR loss at the NMJ. Furthermore, NSG-MG mice strongly mimicked patient MG features. The pathogenic characteristics of the MG thymuses were preserved 2 months after grafting, and large numbers of human cells were found in the periphery.

In the 1990s, some groups attempted to develop humanized MG models in BALB/c Scid mice by grafting MG thymus beneath the renal capsule $(9,24)$ or adoptively infusing PBMCs or peripheral blood lymphocytes into the peritoneum $(10,25-27)$. Although these models displayed anti-AChR Abs, clinical signs were not detectable and human cells were lost rapidly in the mice. The differing levels of immunodeficiency between Scid and NSG mice, as well as dissimilarities in surgical procedures, can account for these differences. To date, NSG mice are the most permissive mice for xenogeneic engraftment (28-31), which likely explains the large number of human cells detected in the spleens of the mice. Of note, in some experiments, the spleens displayed more human cells than murine cells. Furthermore, the site of the graft is likely to be important for the observed differences. Indeed, the subcutaneous location allows the transplantation of relatively large fragments of thymus in comparison with the renal capsule and consequently results in the transfer of more potentially pathogenic cells.

Two months after transplantation, we noticed that the xenogeneic thymus was still active in both CTRL and MG groups. In the MG thymus, we observed the differential expression of genes related to GC 
A

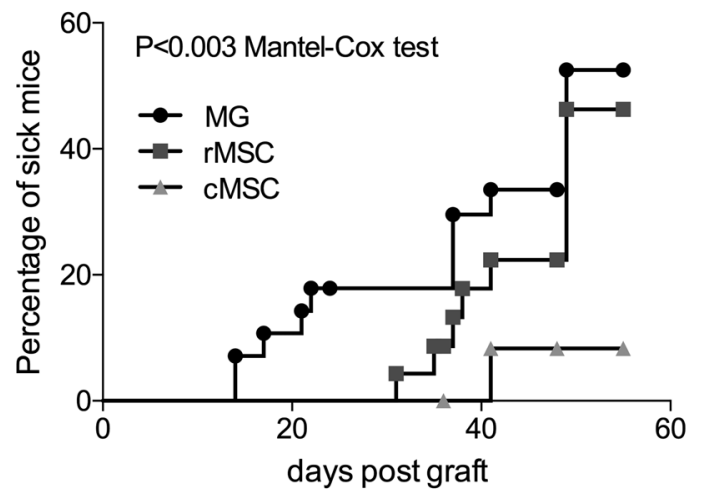

C

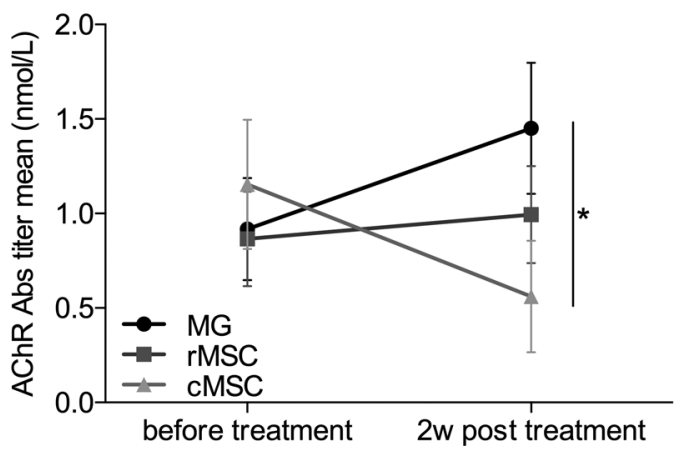

B

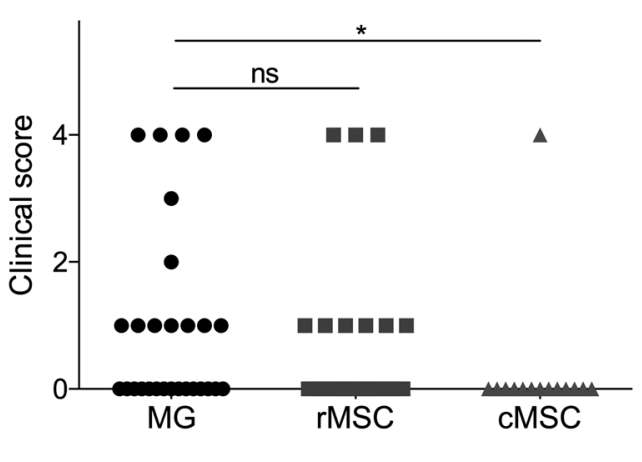

D

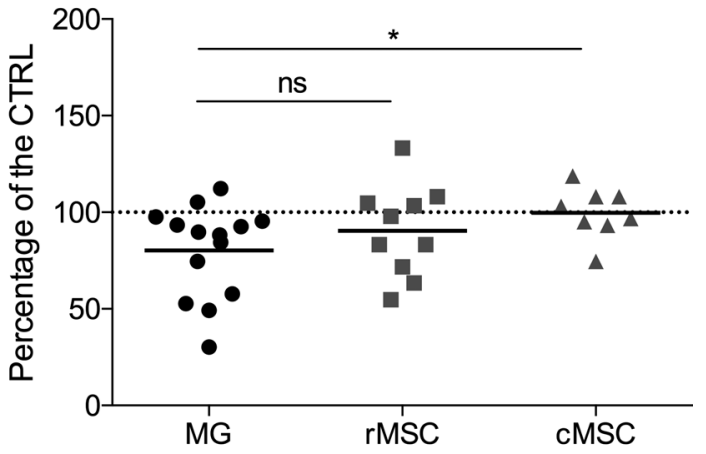

Figure 5. MSC treatment improved myasthenia gravis (MG) features in the NSG-MG model. (A) Mesenchymal stem cell (MSC) treatment reduced MG occurrence. The Kaplan-Meyer curve shows the occurrence of the disease (score $>1$ ) in the MG group (circles, $n=28$ ), in the resting MSC (rMSC) group (squares, $n=23$ ), and in the preconditioned (cMSC) group (triangles, $n=14$ ). The $P$ value was determined using the log-rank (Mantel-Cox) test. (B) MSC treatment reduced MG severity. The histograms represent the mean value \pm SEM of the clinical scores observed for each mouse in each group (MG, $n=28$; rMSC, $n=23$; cMSC, $n=14$ ). (C) MSC treatment reduced acetylcholine receptor-specific (AChR-specific) Abs in the serum. The symbols represent the mean value \pm SEM of the anti-AChR Ab levels observed before and 2 weeks after MSC treatment for the MC group $(n=14)$, for the rMSC group $(n=14)$, and for the cMSC group $(n=10)$. (D) MSC treatment increased muscle endplate AChR content. The AChR contents of the diaphragmatic muscle were measured via ${ }^{125}$ - $\alpha$-bungarotoxin labeling. The data were normalized using the AChR contents of the control (CTRL) group (levels set at 100\%). Each symbol represents the cpm value of 1 mouse, and the bars correspond to the mean values in each group (MG, $n=16 ; \mathrm{rMSC}, n=13$, and cMSC, $n=11$ ). Four to 6 experiments are included. ${ }^{*} P<0.05$ by the Mann-Whitney $t$ test $(\mathbf{B}-\mathbf{D})$. ns, not significant.

initiation (BCL6 was overexpressed compared with CTRL, $P=0.08$, Supplemental Table 1), maintenance (BAFF, Figure 2, and APRIL were overexpressed compared with CTRL, $P=0.07$, Supplemental Table 1) and regulation (IRF4, Figure 2). In the MG thymus, we were also able to detect GCs, suggesting that GCs persisted or that new GCs were generated. Because there is no naive B cell input from the periphery in the NSG-MG mice, it is likely that memory B cells originating from a previous follicular B cell response reinitiate the GCs. The persistence of the GCs can explain the chronicity of the autosensitization mechanism. Thus, the NSG-MG model displayed dynamic and persistent humoral immunity for at least 2 months.

cMSCs as a new therapeutic tool for treating $M G$. Numerous studies have demonstrated the strong and broad immunosuppressive properties of MSCs, making them a promising tool for treating autoimmune diseases (13). Here, we showed that the systemic administration of cMSCs leads to MG improvement. Indeed, MG occurrence and severity were decreased in treated mice. The observations that $\mathrm{AChR}$ Ab levels in the serum are reduced or stabilized and that AChR content at the NMJ is restored are likely explanations for the therapeutic effect. AutoAb-level reduction in the serum is often associated with the recovery of individual MG patients (32).

In the current study, we showed that cMSCs were far more efficient than rMSCs. To date, more than 100 clinical trials have already tested MSCs in immune-mediated diseases, and most (if not all) therapies use resting cells. In a recent work, we showed that supernatants from rMSCs are not able to reduce the 

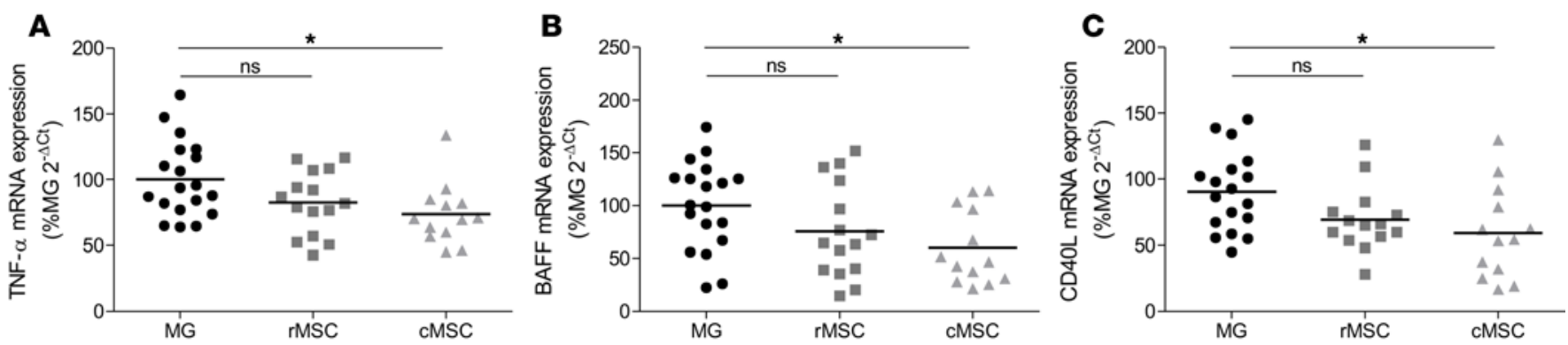

D

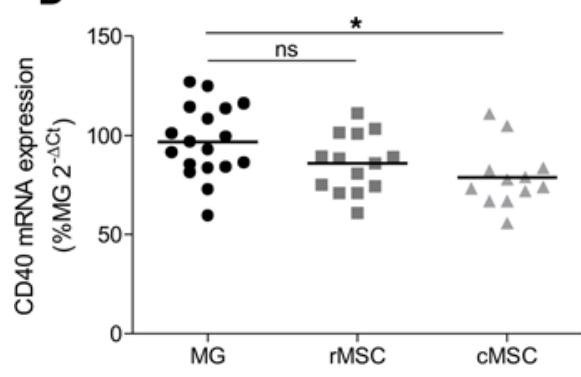

E

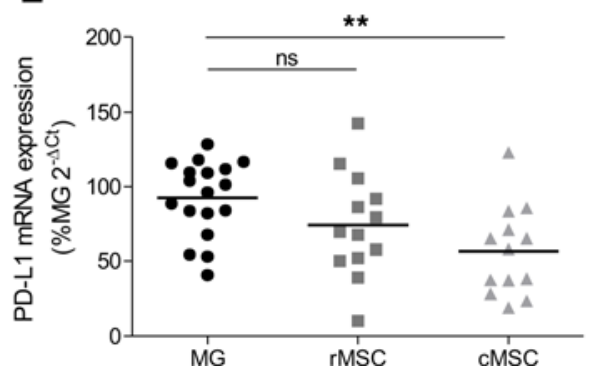

$\mathbf{F}$

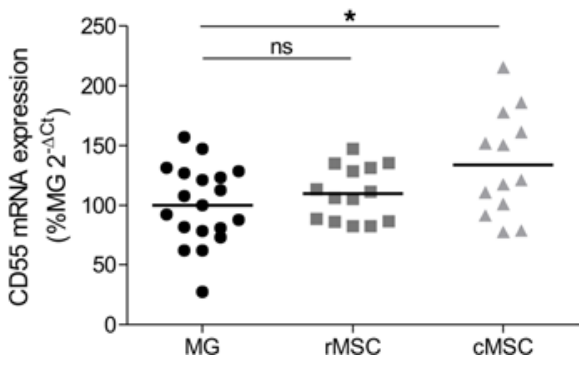

Figure 6. Modulation of gene expression by mesenchymal stem cells (MSCs) in the thymus. TNF- $\alpha, B A F F$, CD40L, CD40, PD-L1, and CD55 mRNA expression was analyzed in the xenogeneic thymus via real-time PCR. The values on the $y$ axis from the myasthenia gravis (MG), resting MCS (rMSC), and preconditioned (cMSC) groups were normalized using the mean $2^{-\Delta c t}$ values of the MG group (levels set at $100 \%$ ). Each symbol represents the normalized $2^{-\Delta C \mathrm{t}}$ value of 1 mouse, and the bars represent the mean values of each group. Four to 6 experiments are included (MC, $n=19, \mathrm{rMSCs}, n=$ 15 , and $\mathrm{cMSC}, n=13) .{ }^{*} P<0.05,{ }^{*} P<0.01$ by 1 -way ANOVA test. ns, not significant.

proliferation of PBMCs, while supernatants from cMSCs are very active (13). In addition, it has been demonstrated that in vitro pretreatment with inflammatory cytokines, such as IFN- $\gamma$, TNF- $\alpha$, IL- 1 , and IL-17, promotes the immunosuppressive capabilities of MSCs in vitro $(17,33,34)$ and that in vitro pretreatment with IFN- $\gamma$ leads to an enhanced therapeutic effect in vivo $(35,36)$. Thus, our conditioning settings provided suitable signals for improving MSC efficiency or facilitating the activation of MSCs in vivo. Actually, MSCs are not immunosuppressive per se; they must be primed (37). This requirement is supported by the findings of Shi et al. (38), who demonstrated that MSCs are most effective when administered after the onset of inflammatory disease. Here, MSCs were infused 2 to 3 weeks after grafting. Because rMSC injection triggered a mild but observable beneficial effect (Figure 5), we suppose that they were licensed in vivo. Despite the higher levels of IL-17, IFN- $\gamma$, and TNF- $\alpha$ within the MG thymus in comparison with the CTRL thymus, rMSCs may not become activated as fully as cMSCs. We also hypothesize that the in vitro activation of MSCs enhanced the migratory potential of the cells.

It has been shown that MSCs home to the site of injury (39). Here, MSC immunosuppressive effects occurred in the thymus rather than in the spleen. Indeed, our extensive analysis of gene expression revealed that the effects of MSCs were essentially restricted to the human thymus, with the exception of mki67. In other words, MSCs acted at the site of autosensitization and inflammation rather than in the periphery, where no signs of inflammation were observed. In support of this hypothesis, in a mouse model of cutaneous delayed-type hypersensitivity, Lim et al. demonstrated that infused MSCs were detected at the site of the immune response, close to GCs in the draining lymph nodes (40).

In our model, a beneficial effect of cMSCs was observed 1.5 to 2 months after their administration, suggesting that either the cells were able to survive in vivo or the cells were able to stably modulate the immune response before dying. Because Yu et al. were able to detect human MSCs that were administrated intravenously 4 weeks earlier in the spleens of EAMG mice (14), we can assume that MSCs may home to the transplanted xenogeneic thymus. A molecular analysis of MSC markers at the end of the experiment (about 2 months) showed that only 1 mouse has a significant level of MSC markers (Supplemental Figure 6), suggesting that cMSCs may home to the xenogeneic thymus. However, it is possible that the timing was not optimal, and a kinetics study is required to increase the chance of finding the MSCs in the grafted thymus consistently. 


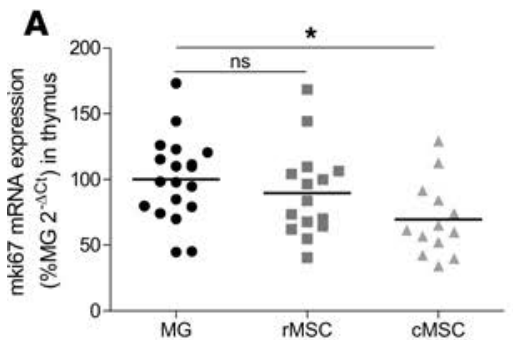

D
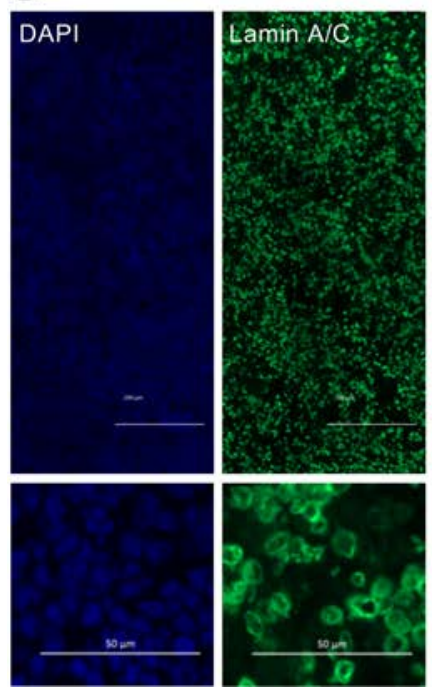

B

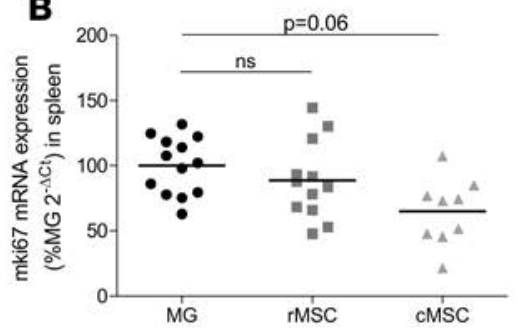

C

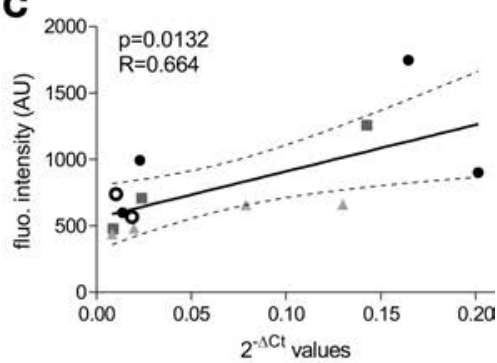

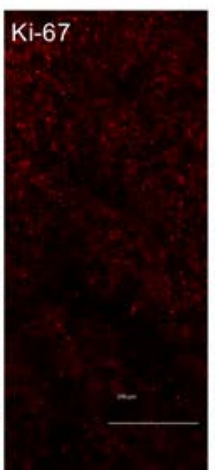

E
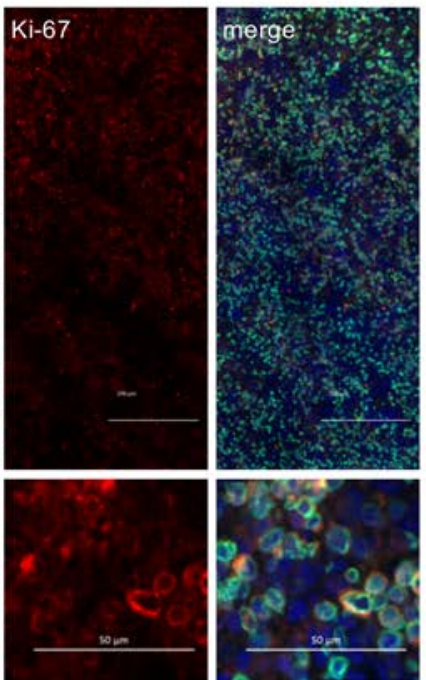
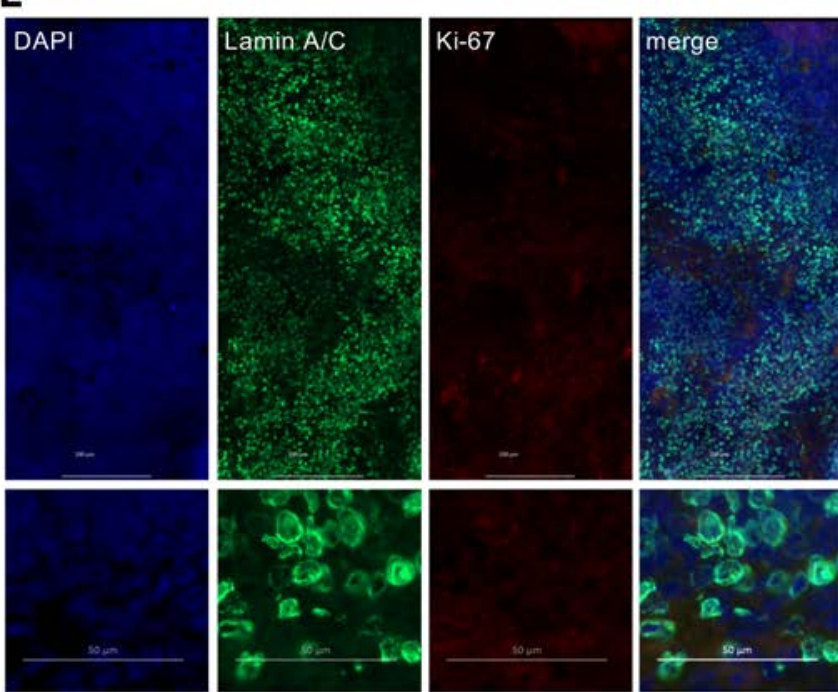

Figure 7. Mesenchymal stem cells (MSCs) inhibited human cell proliferation in the thymus and the spleen. The proliferation status of human cells in the xenogeneic thymus (A) and the spleen (B) was analyzed at the mRNA level via real-time PCR. The values on the $y$ axis (\%MC $\left.2^{-\triangle C t}\right)$ for the resting MSC (rMSC) and preconditioned MCS (cMSC) groups were normalized using the mean $2^{-\Delta c t}$ values of the MG group (levels set at $\left.100 \%\right)$. Each symbol represents the $2^{-\Delta c t}$ value of 1 mouse, and the bars represent the mean values in the myasthenia gravis (MG) and cMSC groups. Four to 6 experiments are included (MG, $n=19$ mice, rMSC, $n=15$ and cMSC, $n=13$ mice). $P$ values were determined using the 1 -way ANOVA test. ${ }^{*} P<0.05$. (C-E) The proliferation status of human cells in the spleen was also analyzed at the protein level via IHC. (C) mki67 mRNA expression correlated with KI-67 fluorescence intensity. Each symbol represents 1 mouse, and 2 experiments are included: MG, black circles, $n=4$; rMSC, dark gray squares, $n=3$; CMSC, light gray triangles, $n=4$; CTRL, open circles, $n=2$. $P$ values were determined using the linear regression test. ( $\mathbf{D}$ and $\mathbf{E}$ ) Upper panels, mosaic with nearly all spleen sections; lower panels, representative image showing human cells (lamin A/C-positive cells, in green) and proliferating cells (KI-67-positive cells, in red) among all splenocytes (DAPI positive cells, in blue) in the MG group (D) and the cMSC group (E). Scale bars: $200 \mu \mathrm{m}$ (top panels) and $50 \mu \mathrm{m}$ (bottom panels) in $\mathbf{D}$ and $\mathbf{E}$.

Mechanisms of action of cMSCs. Our data point to a potentially new mechanism of action of cMSCs via the upregulation of CD55 (DAF) that regulates the immune system via complement-dependent and -independent mechanisms $(41,42)$. Because DAF deficiency was previously associated with autoimmunity, including MG (43), and was conversely shown to augment susceptibility to EAMG (44, 45), CD55 mRNA augmentation in cMSC-treated mice may partially explain the observed MG improvement in our NSG-MG model. It has been shown that DAF expression at the NMJ likely limits MAC damage in MG $(43,44)$. Interestingly, DAF was also shown to be involved in T cell activation directly or via its ligand CD97 (41), suggesting that DAF augmentation might be beneficial, not only by limiting MAC damage but also by modulating $\mathrm{T}$ cell activation in the thymus and lymphoid organs. Soltys et al. showed increased

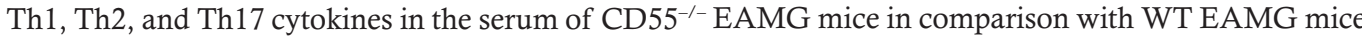
(44). Our data showing that upregulation of CD55 after cMSC therapy paralleled the decreased expression of TNF- $\alpha$ are compatible with an antiinflammatory effect of CD55 signaling (42).

In addition to this potentially new mechanism, we observed that genes of TNF family ligands (TNF- $\alpha$, BAFF, CD40L, and CD40), which were increased in the MG thymus, decreased to normal values in the cMSC-treated groups compared with the untreated group. These genes play a central role in inflammation and autoimmunity $(46,47)$. In MG disease, TNF- $\alpha$ is increased in the thymus and the sera (5), and BAFF levels are higher in MG patients than in CTRL subjects (48-50). In addition, TNF- $\alpha$ is overexpressed during EAMG 


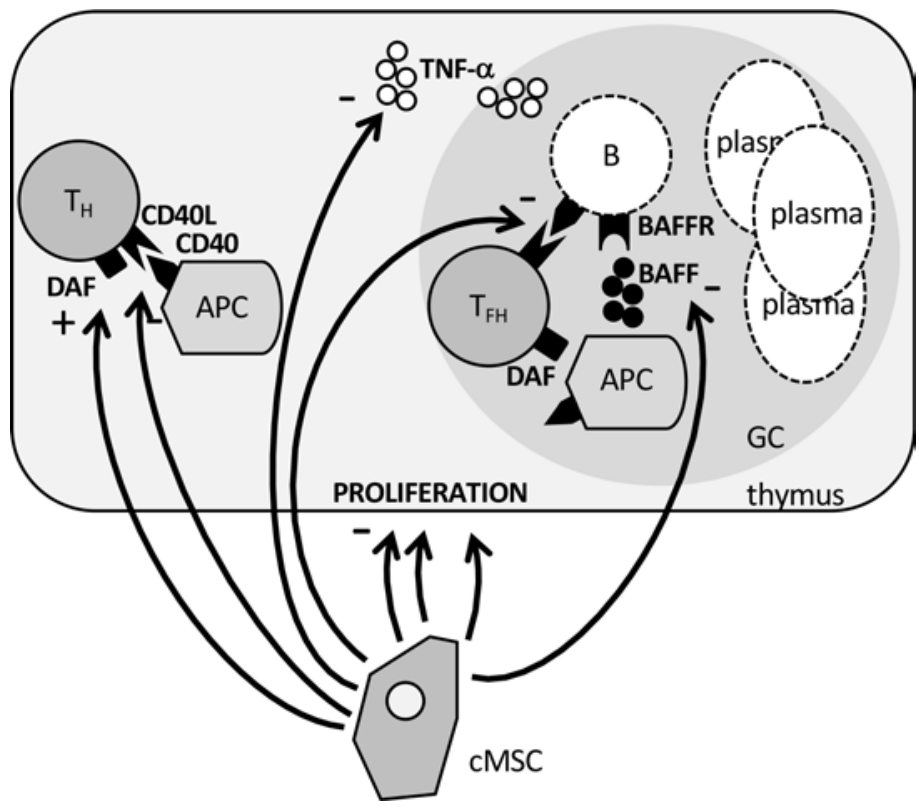

Figure 8. Schematic diagram summarizing the immunosuppressive effects of cMSCs in the NSG-MG model. The inhibition of TNF ligand (i.e., TNF- $\alpha$, BAFF, and CD4OL) mRNA expression in the human thymus may occur in T cells or antigen-presenting cells (APCs). In addition to antiinflammatory effects, preconditioned mesenchymal stem cells (cMSCs) modulate accessory molecules. The mRNA expression of the costimulatory molecule CD40 is reduced and the mRNA expression of the coinhibitory molecule CD55 is augmented in comparison with the untreated group. We hypothesize that by suppressing inflammation and modulating the immune response, cMSCs prevent the myasthenogenic reaction. In addition, we propose that the inhibition of the CD40CD40L interaction in B cells may explain the observed reduction of acetylcholine receptor-specific Abs in the serum of treated mice. In the thymus and the spleen of cMSC-treated mice, cellular proliferation is decreased. NSG-MG, immunodeficient NSG mice with subcutaneously grafted thymic myasthenia gravis fragments; $\mathrm{T}_{\mathrm{FH}}, \mathrm{T}$ follicular helper cell; GC, germinal center.

development $(51,52)$. Furthermore, Im et al. demonstrated that CD40L blockade suppresses EAMG (53). Thus, our findings are in agreement with the literature data highlighting a central role of these molecules in MG pathogenesis, and demonstrate that the cMSC active therapy restored normal levels of these molecules.

Our results are also consistent with several in vivo and in vitro studies on the mechanisms of action of MSCs. Indeed, MSCs were shown to decrease CD40L in PBMCs in immune thrombocytopenia patients (54) and to decrease BAFF plasma levels in responding graft-versus-host disease patients (55). MSCs were also shown to inhibit TNF- $\alpha$ in the central nervous system in experimental autoimmune encephalomyelitis (56) and the uterine tissue in an autoimmune preeclampsia model (57). Furthermore, in vitro studies demonstrated that MSCs could promote tolerogenic DCs by reducing proinflammatory cytokines, such as TNF- $\alpha(58,59)$, and costimulatory molecules, including CD40 $(58,60)$. Although our data do not show a strong reduction by $\mathrm{cMSC}$ of many proinflammatory cytokines, they show a significant reduction in TNF- $\alpha$, which plays a key role in MG pathogenesis (5).

Finally, cMSCs had a convincing effect on B cell-related molecules. Both the CD40-CD40L and PD1PDL1 interactions, which trigger survival signaling to follicular B cells, are required for GC formation and the maintenance of GC B cells (61), and BAFF is a potent B cell activator involved in B cell proliferation (20). Thus, cMSCs could improve MG by inhibiting accessory molecules involved in B cell activation. The BAFF mRNA reduction in the thymus could give an explanation for the B cell decrease (reduction in CD20) observed in the thymus, which could result in diminished production of AChR Abs and improvement of MG.

Conclusion. In conclusion, in the NSG-MG model, cMSCs displayed antiinflammatory and immunosuppressive effects primarily in the thymus, leading to MG improvement. Figure 8 summarizes the putative effects of cMSCs in MG. Interestingly, those effects combine several current MG therapeutic strategies, including the anti-BAFF Ab (belimumab; ClinicalTrial.gov identifier: NCT01480596), the anti-C5 Ab, which targets a component of MAC (eculizumab; ClinicalTrial.gov identifier: NCT00727194) and antiproliferative agents (methotrexate, azathioprine). Because a single injection of cMSCs was effective for at least 1 month, this approach that combines several therapeutic effects and acts at different levels of pathology represents a promising cell therapy for $\mathrm{MG}$.

\section{Methods}

Patients. The MG thymuses used in this study were not associated with thymoma. The clinical details of the MG patients are summarized in Table 1. The CTRL thymuses, which were obtained from newborn patients undergoing corrective heart surgery, showed no phenotypic or histological abnormalities. Thymectomy was performed at the Centre Chirurgical Marie Lannelongue or the Hôpital Civil de Strasbourg. All thymuses removed from the patients were immediately transferred to RPMI medium at $4^{\circ} \mathrm{C}$ and were processed within 24 hours after thymectomy. 
Animals. NOD-scid IL-2R $\gamma^{\text {null }}$ (NSG) mice were obtained from Charles River Laboratories. The mice were bred in our animal facilities under specific pathogen-free conditions and were used between 8 and 14 weeks of age.

The mice were weighed weekly and bled 2 times per month from the superficial temporal vein (mandibular). Serum was collected and stored frozen $\left(-20^{\circ} \mathrm{C}\right)$. Six to 8 weeks after transplantation, the animals were euthanized by cervical dislocation or $\mathrm{CO}_{2}$ inhalation. The diaphragms, xenogeneic thymuses, spleens, and bone marrow were removed and fixed, frozen, or used fresh.

Xenogenic thymus transplantation. After the removal of the capsule, the human thymic tissue was cut with scissors in Hanks buffer (Invitrogen) into several fragments of approximately $5 \mathrm{~mm}$ per side. Three of these fragments (randomly chosen) were transplanted subcutaneously into the lower backs of anesthetized ( $80 \mathrm{mg} / \mathrm{kg}$ body weight ketamine and $4 \mathrm{mg} / \mathrm{kg}$ body weight xylazine) NSG mice. For each thymus, 6 to 15 mice were transplanted, based on the amount of tissue available. All surgical procedures were performed under a laminar flow hood and using aseptic conditions.

Clinical scoring. The MG-like clinical score was assessed by observing mouse behavior and was graded on a scale of 0 to 4 , as follows: score 0 , no sign; score 1 , abnormal movements (walking with head and tail down); score 2, reduced motility; score 3, hunched posture; score 4, paralysis, dehydration or death. The animals were considered to be sick when they reached score 1 (i.e., when they displayed altered movements).

MSC culture, priming, and injection. MSCs were isolated from human adipose tissues and cultured and characterized as described previously (18).

In vitro MSC priming consists of a 3-day coculture with allogeneic PBMCs. Briefly, MSCs were seeded into 6-well plates in 1:1 DMEM/F12(HAM) (Biological Industries) supplemented with 10\% FCS (Eurobio), $1 \%$ penicillin/streptomycin and 1\% L-glutamine (Gibco). Allogeneic PBMCs (2:1 ratio) or DMEM/ F12 medium alone were added to the MSC culture when the cells reached approximately $90 \%$ confluence. The PBMCs were separated from the adherent MSCs using Transwell membrane cell culture inserts $(1 \mu \mathrm{m}$ pore size, Becton Dickinson), which prevented cell contact but allowed the diffusion of soluble mediators (62). PBMCs were obtained from venous blood from healthy volunteer donors (Etablissement Français du Sang, Rungis, France) using the Lymphoprep density gradient centrifugation protocol (Axis-Shield). After 3 days of coculture, the insert containing the PBMCs was removed, and the adherent MSCs were detached using a $0.25 \%$ trypsin and $0.01 \%$ EDTA solution for 10 minutes and washed 3 times in PBS. Then, $2 \times 10^{5}$ to $1 \times 10^{6}$ cells were injected intravenously into mice 2 to 3 weeks after MG thymus transplantation.

Flow cytometry. FACS analyses were performed on cells from the spleen, blood, and bone marrow of grafted animals. The spleens were dissociated mechanically in PBS containing 3\% FCS to isolate splenocytes. Bone marrow cells were collected by flushing the femurs and tibiae with 3\% FCS in PBS using a 26-gauge needle. For spleen and blood samples, erythrocytes were removed via incubation with a $0.84 \% \mathrm{NH}_{4} \mathrm{Cl}$ solution for 1 minute and BD Pharm Lyse for 10 minutes (BD Biosciences), respectively. Single cells were then filtered $(70 \mu \mathrm{m})$, washed twice, and stained for 30 minutes on ice with anti-CD45, -CD4, -CD8, and -CD19 Abs and with LiveDead (to assay cell viability, Life Technologies) (see details in Supplemental Table 1). The cells were acquired on a FACSVerse system (BD Biosciences) and analyzed using FlowJo software (Tree Star).

Immunochemistry of mouse spleen and human thymus sections. Cryosections $(7 \mu \mathrm{m})$ of mouse spleens and human thymuses were collected on superfrost slides (Thermo Fisher Scientific), fixed in ice-cold acetone for 20 minutes, and blocked in a solution of 3\% FCS in PBS to avoid nonspecific binding. The sections were first stained at room temperature for 2 hours with primary Abs directed against human cytokeratin, fibronectin, CD21, CD4, CD8, KI-67, and lamin A/C. An Ab specific for human lamin A/C was used to visualize the human cells in the mouse spleen, as it binds to human but not mouse nuclei. After 3 washes in PBS, the sections were stained at room temperature for 1 hour with the secondary Abs. After 3 washes in PBS, the sections were then stained at room temperature for 5 minutes with DAPI (Life Technologies). The characteristics of the Abs are detailed in Supplemental Table 2. The slides were mounted in Faramount fluorescent mounting media (Dako). Images were acquired with a Zeiss Axio Observer Z1 inverted microscope with a $10 \times$ eyepiece and a $20 \times$ objective, using a Zeiss AxioCam MRm camera. The acquisition software was Axiovision (Zeiss).

Detection of human anti-AChR Abs. AChR-specific human Abs were detected in mouse serum via classical RIA, as described previously (63). Briefly, crude extracts of human muscles complexed with ${ }^{125} \mathrm{I}$ - $\alpha$-bungarotoxin ( $\alpha$-BGT) were incubated with $10 \mu \mathrm{l}$ of mouse serum. Abs were then precipitated with 
anti-human IgG, using $2.5 \mu$ of normal human serum as carrier IgG. Several time points were analyzed for each mouse, and because the kinetics were not identical for all mice, the results were expressed as the highest level of anti-AChR for each mouse.

$R N A$ extraction and real-time PCR ( $q P C R$ ) analysis. Frozen mouse spleens and human thymuses were disrupted with a FastPrep apparatus (QBiogen), and total RNA was extracted with TRIzol (Life Technologies), according to the manufacturer's instruction. One microgram of RNA was reverse transcribed for 1 hour at $42^{\circ} \mathrm{C}$ using AMV (Roche Applied Science) with oligo-dT (Invitrogen).

We analyzed the expression of a panel of 57 genes involved in immune cell response, inflammation, or cell cycle, as well as 3 housekeeping genes (Supplemental Table 3). The primer sets were provided by realtimeprimers.com. The qPCR reaction was performed on a 1,536-well Light Cycler apparatus (Roche), in which samples were set with a Bravo Automated Liquid Handling system (Agilent). $\Delta \mathrm{Ct}$ values were determined as the difference between the cycle threshold $(\mathrm{Ct})$ value of the gene of interest and the mean Ct value of the 3 most stable housekeeping genes: GUSB, PPIA, and GAPD. The data are expressed as $2^{-\Delta \mathrm{Ct}}$ values or normalized using the mean $2^{-\Delta \mathrm{Ct}}$ values of the CTRL group (levels set at $100 \%$ ).

Endplate AChR quantification. AChR quantification was assessed at the diaphragmatic muscular endplate using specific $\alpha$-BGT binding and based on a previously described method (10). Briefly, the diaphragms were carefully harvested from grafted mice and fixed in a solution of $4 \%$ formaldehyde in PBS (Sigma-Aldrich). Three to 5 biopsies of 2-mm diameter (skin biopsy punch, Help Médical) were collected along the NMJ and AChR was localized using the histochemical Koelle and Friedenwald reaction that characterizes AChE activity (64). Each biopsy was first incubated at room temperature for half an hour in a solution of $5 \%$ FCS in PBS, washed 3 times in a solution of $0.5 \%$ FCS in PBS, and then labeled with 0.1 $\mu \mathrm{Ci}$ of ${ }^{125} \mathrm{I}-\alpha$-BGT (i.e., $4 \mu \mathrm{Ci} / \mathrm{ml}$, specific activity $10-20 \mu \mathrm{Ci} / \mu \mathrm{g}$, PerkinElmer) at room temperature for 15 minutes. The biopsies were washed 3 times in a large volume of PBS at room temperature for at least 30 minutes, and radioactivity was measured with a LB 2111 gamma counter (Berthold Technologies, Bad Wildbad, Germany). For each experiment, the cpm values of the MG group were normalized using the mean cpm values of the CTRL group (level set at 100\%).

Statistics. Differences between independent experimental groups were analyzed using GraphPad Prism 6 software. When more than 2 groups were compared, the 1-way ANOVA test was used. When 2 groups were compared, a nonparametric Mann-Whitney $t$ test was used. When appropriate, the linear regression test or the log-rank (Mantel-Cox) test was used. All differences were calculated using a 2-tailed test. The test is specified in the figure legend. Statistical significance was recognized at $P$ less than 0.05 . In all figures, the significance is displayed as asterisks, as follows: ${ }^{*} P<0.05,{ }^{* *} P<0.01,{ }^{* * *} P<0.001, * * * * P<0.0001$.

Study approval. This human study was approved by the local ethics committee (CCP, authorization number ID RCB 2010-A00250-39), and informed consent was obtained from the patient or the patient's legal representative.

The animal study was approved by the French Ministry of Agriculture committee for the animal used (authorization number 02622.2). All animals were handled according to the Animal Care and Use of Laboratory Animal guidelines of the French Ministry of Research under study approval number 02638-02.

\section{Author contributions}

MS designed and performed the experiments, analyzed the data, and interpreted the results. MM, MR, $\mathrm{ND}$, and MA contributed to some experiments in mice. JB and DG assisted with the immunofluorescence experiments. FT provided logistical support related to thymuses and clinical data. EF and NS provided human thymic tissues. CS and TB performed the titration of the anti-AChR Abs. SBA was involved in all aspects of the study, including study design, data analysis, and the interpretation of the results. MS and SBA wrote the manuscript. All authors discussed the results and commented on the manuscript.

\section{Acknowledgments}

We would like to thank Eric Noe from the Institute for Brain and Spinal Cord (ICM) for his help with experiments involving radioactive compounds. We also thank Yannick Marie and Delphine Bouteiller from the genotyping and sequencing ICM platform for the PCR experiments. We are very grateful to Jossi Itskovitz-Eldor and Liron Eldor (RamBam Hospital) for making the human MSCs available. We would like to acknowledge UMS28-Phénotypage du petit animal for technical advice and excellent animal care. 
This work was supported by FIGHT-MG (HEALTH-2009-242-210) grants from the European Community and a grant from the Association Française Contre Les Myopathies, which were obtained by S. Berrih-Aknin.

Address correspondence to: Sonia Berrih-Aknin, Université Pierre et Marie Curie - Paris VI, INSERM U974- Hôpital La Pitié Salpêtrière, 105 Bd de l'Hôpital, 75013 Paris, France. Phone: 00.33.0.1.40.77.81.28; E-mail: sonia.berrih-aknin@upmc.fr.

1. Meriggioli MN. Myasthenia gravis with anti-acetylcholine receptor antibodies. Front Neurol Neurosci. 2009;26:94-108.

2. Vrolix K, et al. The auto-antigen repertoire in myasthenia gravis. Autoimmunity. 2010;43(5-6):380-400.

3. Howard FM, Lennon VA, Finley J, Matsumoto J, Elveback LR. Clinical correlations of antibodies that bind, block, or modulate human acetylcholine receptors in myasthenia gravis. Ann N Y Acad Sci. 1987;505:526-538.

4. Berrih-Aknin S, Ragheb S, Le Panse R, Lisak RP. Ectopic germinal centers, BAFF and anti-B-cell therapy in myasthenia gravis. Autoimmun Rev. 2013;12(9):885-893.

5. Gradolatto A, et al. Both Treg cells and Tconv cells are defective in the myasthenia gravis thymus: roles of IL-17 and TNF- $\alpha$ J Autoimmun. 2014;52:53-63.

6. Berrih-Aknin S, Le Panse R. Myasthenia gravis: a comprehensive review of immune dysregulation and etiological mechanisms. J Autoimmun. 2014;52:90-100.

7. Sieb JP. Myasthenia gravis: an update for the clinician. Clin Exp Immunol. 2014;175(3):408-418

8. Meinl E, Klinkert WE, Wekerle H. The thymus in myasthenia gravis. Changes typical for the human disease are absent in experimental autoimmune myasthenia gravis of the Lewis rat. Am J Pathol. 1991;139(5):995-1008.

9. Schönbeck S, Padberg F, Hohlfeld R, Wekerle H. Transplantation of thymic autoimmune microenvironment to severe combined immunodeficiency mice. A new model of myasthenia gravis. J Clin Invest. 1992;90(1):245-250.

10. Aissaoui A, et al. Prevention of autoimmune attack by targeting specific T-cell receptors in a severe combined immunodeficiency mouse model of myasthenia gravis. Ann Neurol. 1999;46(4):559-567.

11. Le Blanc K, Ringdén O. Immunomodulation by mesenchymal stem cells and clinical experience. J Intern Med. 2007;262(5):509-525.

12. Madrigal M, Rao KS, Riordan NH. A review of therapeutic effects of mesenchymal stem cell secretions and induction of secretory modification by different culture methods. J Transl Med. 2014;12:260.

13. Ben-Ami E, Berrih-Aknin S, Miller A. Mesenchymal stem cells as an immunomodulatory therapeutic strategy for autoimmune diseases. Autoimmun Rev. 2011;10(7):410-415.

14. Yu J, et al. Intravenous administration of bone marrow mesenchymal stem cells benefits experimental autoimmune myasthenia gravis mice through an immunomodulatory action. Scand J Immunol. 2010;72(3):242-249.

15. Kong QF, et al. BM stromal cells ameliorate experimental autoimmune myasthenia gravis by altering the balance of Th cells through the secretion of IDO. Eur J Immunol. 2009;39(3):800-809.

16. Krampera M, et al. Role for interferon-gamma in the immunomodulatory activity of human bone marrow mesenchymal stem cells. Stem Cells. 2006;24(2):386-398.

17. Ren G, et al. Mesenchymal stem cell-mediated immunosuppression occurs via concerted action of chemokines and nitric oxide. Cell Stem Cell. 2008;2(2):141-150.

18. Ben-Ami E, Miller A, Berrih-Aknin S. T cells from autoimmune patients display reduced sensitivity to immunoregulation by mesenchymal stem cells: role of IL-2. Autoimmun Rev. 2014;13(2):187-196.

19. Berrih-Aknin S, et al. The role of the thymus in myasthenia gravis: immunohistological and immunological studies in 115 cases. Ann N Y Acad Sci. 1987;505:50-70.

20. Schneider P, et al. BAFF, a novel ligand of the tumor necrosis factor family, stimulates B cell growth. J Exp Med. 1999;189(11):1747-1756.

21. Avery DT, et al. BAFF selectively enhances the survival of plasmablasts generated from human memory B cells. J Clin Invest 2003;112(2):286-297.

22. Ochiai $\mathrm{K}$, et al. Transcriptional regulation of germinal center B and plasma cell fates by dynamical control of IRF4. Immunity. 2013;38(5):918-929.

23. Berrih-Aknin S. Myasthenia gravis: paradox versus paradigm in autoimmunity. J Autoimmun. 2014;52:1-28.

24. Spuler S, Sarropoulos A, Marx A, Hohlfeld R, Wekerle H. Thymoma-associated myasthenia gravis. Transplantation of thymoma and extrathymomal thymic tissue into SCID mice. Am J Pathol. 1996;148(5):1359-1365.

25. Wang ZY, Karachunski PI, Howard JF, Conti-Fine BM. Myasthenia in SCID mice grafted with myasthenic patient lymphocytes: role of CD4+ and CD8 ${ }^{+}$cells. Neurology. 1999;52(3):484-497.

26. Martino G, et al. The human-severe combined immunodeficiency myasthenic mouse model: a new approach for the study of myasthenia gravis. Ann Neurol. 1993;34(1):48-56.

27. Vassilev T, et al. Normal human immunoglobulin suppresses experimental myasthenia gravis in SCID mice. Eur J Immunol. 1999;29(8):2436-2442.

28. Lepus CM, et al. Comparison of human fetal liver, umbilical cord blood, and adult blood hematopoietic stem cell engraftment in NOD-scid/gammac-/-, Balb/c-Rag1-/-gammac-/-, and C.B-17-scid/bg immunodeficient mice. Hum Immunol. 2009;70(10):790-802.

29. McDermott SP, Eppert K, Lechman ER, Doedens M, Dick JE. Comparison of human cord blood engraftment between immunocompromised mouse strains. Blood. 2010;116(2):193-200.

30. Stoddart CA, et al. Superior human leukocyte reconstitution and susceptibility to vaginal HIV transmission in humanized NOD-scid IL-2R $\gamma(-/-)$ (NSG) BLT mice. Virology. 2011;417(1):154-160.

31. Favre D, et al. HIV disease progression correlates with the generation of dysfunctional naive CD8(low) T cells. Blood. 
2011;117(7):2189-2199.

32. Seybold ME, Lindstrom JM. Patterns of acetylcholine receptor antibody fluctuation in myasthenia gravis. Ann N Y Acad Sci. 1981;377:292-306.

33. Marigo I, Dazzi F. The immunomodulatory properties of mesenchymal stem cells. Semin Immunopathol. 2011;33(6):593-602.

34. Han X, et al. Interleukin-17 enhances immunosuppression by mesenchymal stem cells. Cell Death Differ. 2014;21(11):1758-1768.

35. Polchert D, et al. IFN-gamma activation of mesenchymal stem cells for treatment and prevention of graft versus host disease. Eur J Immunol. 2008;38(6):1745-1755.

36. Duijvestein $M$, et al. Pretreatment with interferon- $\gamma$ enhances the therapeutic activity of mesenchymal stromal cells in animal models of colitis. Stem Cells. 2011;29(10):1549-1558.

37. Krampera M. Mesenchymal stromal cell 'licensing': a multistep process. Leukemia. 2011;25(9):1408-1414.

38. Shi Y, Su J, Roberts AI, Shou P, Rabson AB, Ren G. How mesenchymal stem cells interact with tissue immune responses. Trends Immunol. 2012;33(3):136-143.

39. Yagi H, et al. Mesenchymal stem cells: Mechanisms of immunomodulation and homing. Cell Transplant. 2010;19(6):667-679.

40. Lim JH, et al. Immunomodulation of delayed-type hypersensitivity responses by mesenchymal stem cells is associated with bystander T cell apoptosis in the draining lymph node. J Immunol. 2010;185(7):4022-4029.

41. Clarke EV, Tenner AJ. Complement modulation of T cell immune responses during homeostasis and disease. J Leukoc Biol. 2014;96(5):745-756.

42. Toomey CB, Cauvi DM, Pollard KM. The role of decay accelerating factor in environmentally induced and idiopathic systemic autoimmune disease. Autoimmune Dis. 2014;2014:452853.

43. Heckmann JM, Uwimpuhwe H, Ballo R, Kaur M, Bajic VB, Prince S. A functional SNP in the regulatory region of the decayaccelerating factor gene associates with extraocular muscle pareses in myasthenia gravis. Genes Immun. 2010;11(1):1-10.

44. Soltys J, Halperin JA, Xuebin Q. DAF/CD55 and protectin/CD59 modulate adaptive immunity and disease outcome in experimental autoimmune myasthenia gravis. J Neuroimmunol. 2012;244(1-2):63-69.

45. Lin F, Kaminski HJ, Conti-Fine BM, Wang W, Richmonds C, Medof ME. Markedly enhanced susceptibility to experimental autoimmune myasthenia gravis in the absence of decay-accelerating factor protection. J Clin Invest. 2002;110(9):1269-1274.

46. Aggarwal BB, Gupta SC, Kim JH. Historical perspectives on tumor necrosis factor and its superfamily: 25 years later, a golden journey. Blood. 2012;119(3):651-665.

47. Sethi G, Sung B, Kunnumakkara AB, Aggarwal BB. Targeting TNF for treatment of cancer and autoimmunity. Adv Exp Med Biol. 2009;647:37-51.

48. Kim JY, et al. Serum BAFF expression in patients with myasthenia gravis. J Neuroimmunol. 2008;199(1-2):151-154.

49. Ragheb S, Lisak R, Lewis R, Van Stavern G, Gonzales F, Simon K. A potential role for B-cell activating factor in the pathogenesis of autoimmune myasthenia gravis. Arch Neurol. 2008;65(10):1358-1362.

50. Scuderi F, Alboini PE, Bartoccioni E, Evoli A. BAFF serum levels in myasthenia gravis: effects of therapy. J Neurol. 2011;258(12):2284-2285.

51. Duan RS, Wang HB, Yang JS, Scallon B, Link H, Xiao BG. Anti-TNF-alpha antibodies suppress the development of experimental autoimmune myasthenia gravis. J Autoimmun. 2002;19(4):169-174.

52. Wang HB, Li H, Shi FD, Chambers BJ, Link H, Ljunggren HG. Tumor necrosis factor receptor-1 is critically involved in the development of experimental autoimmune myasthenia gravis. Int Immunol. 2000;12(10):1381-1388.

53. Im SH, Barchan D, Maiti PK, Fuchs S, Souroujon MC. Blockade of CD40 ligand suppresses chronic experimental myasthenia gravis by down-regulation of Th1 differentiation and up-regulation of CTLA-4. J Immunol. 2001;166(11):6893-6898.

54. Ma L, et al. Immunosuppressive function of mesenchymal stem cells from human umbilical cord matrix in immune thrombocytopenia patients. Thromb Haemost. 2012;107(5):937-950.

55. Peng Y, et al. Mesenchymal stromal cells infusions improve refractory chronic graft versus host disease through an increase of $\mathrm{CD}^{+}$regulatory B cells producing interleukin 10. Leukemia. 2015;29(3):636-646.

56. Wang J, et al. Interleukin-27 suppresses experimental autoimmune encephalomyelitis during bone marrow stromal cell treatment. J Autoimmun. 2008;30(4):222-229.

57. Liu L, et al. Mesenchymal stem cells ameliorate Th1-induced pre-eclampsia-like symptoms in mice via the suppression of TNF- $\alpha$ expression. PLoS ONE. 2014;9(2):e88036.

58. Zhang B, et al. Mesenchymal stem cells induce mature dendritic cells into a novel Jagged-2-dependent regulatory dendritic cell population. Blood. 2009;113(1):46-57.

59. Liu X, et al. Mesenchymal stem/stromal cells induce the generation of novel IL-10-dependent regulatory dendritic cells by SOCS3 activation. J Immunol. 2012;189(3):1182-1192.

60. Zhang W, et al. Effects of mesenchymal stem cells on differentiation, maturation, and function of human monocyte-derived dendritic cells. Stem Cells Dev. 2004;13(3):263-271.

61. Nera KP, Kyläniemi MK, Lassila O. Regulation of B cell to plasma cell transition within the follicular B cell response. Scand J Immunol. 2015;82(3):225-234.

62. Hof-Nahor I, et al. Human mesenchymal stem cells shift CD $8^{+} \mathrm{T}$ cells towards a suppressive phenotype by inducing tolerogenic monocytes. J Cell Sci. 2012;125(Pt 19):4640-4650.

63. Gur-Wahnon D, Mizrachi T, Wald-Altman S, Al-Roof Higazi A, Brenner T. Tissue plasminogen activator involvement in experimental autoimmune myasthenia gravis: aggravation and therapeutic potential. J Autoimmun. 2014;52:36-43.

64. Karnovsky MJ, Roots L. A “direct-coloring” thiocholine method for cholinesterases. J Histochem Cytochem. 1964;12:219-221.

65. Gajdos P, Sharshar T, Chevret S. Standards of measurements in myasthenia gravis. Ann N Y Acad Sci. 2003;998:445-452.

66. Barohn RJ. Standards of measurements in myasthenia gravis. Ann N Y Acad Sci. 2003;998:432-439.

67. Truffault F, de Montpreville V, Eymard B, Sharshar T, Le Panse R, Berrih-Aknin S. Thymic germinal centers and corticosteroids in myasthenia gravis: an immunopathological study in 1035 cases and a critical review. Clin Rev Allergy Immunol. 2017;52(1):108-124 